\title{
Systems pharmacology identifies drug targets for Stargardt disease-associated retinal degeneration
}

\author{
Yu Chen, ${ }^{1}$ Grazyna Palczewska, ${ }^{2}$ Debarshi Mustafi, ${ }^{1}$ Marcin Golczak, ${ }^{1}$ Zhiqian Dong, ${ }^{2}$ \\ Osamu Sawada, ${ }^{3}$ Tadao Maeda, ${ }^{3}$ Akiko Maeda, ${ }^{1,3}$ and Krzysztof Palczewski ${ }^{1}$ \\ 1Department of Pharmacology, School of Medicine, Case Western Reserve University, Cleveland, Ohio, USA. ${ }^{2}$ Polgenix, Inc., Cleveland, Ohio, USA. \\ ${ }^{3}$ Department of Ophthalmology, School of Medicine, Case Western Reserve University, Cleveland, Ohio, USA.
}

\begin{abstract}
A systems pharmacological approach that capitalizes on the characterization of intracellular signaling networks can transform our understanding of human diseases and lead to therapy development. Here, we applied this strategy to identify pharmacological targets for the treatment of Stargardt disease, a severe juvenile form of macular degeneration. Diverse GPCRs have previously been implicated in neuronal cell survival, and crosstalk between GPCR signaling pathways represents an unexplored avenue for pharmacological intervention. We focused on this receptor family for potential therapeutic interventions in macular disease. Complete transcriptomes of mouse and human samples were analyzed to assess the expression of GPCRs in the retina. Focusing on adrenergic (AR) and serotonin (5-HT) receptors, we found that adrenoceptor $\alpha 2 \mathrm{C}$ (Adra2c) and serotonin receptor $2 \mathrm{a}(\mathrm{Htr} 2 \mathrm{a})$ were the most highly expressed. Using a mouse model of Stargardt disease, we found that pharmacological interventions that targeted both GPCR signaling pathways and adenylate cyclases (ACs) improved photoreceptor cell survival, preserved photoreceptor function, and attenuated the accumulation of pathological fluorescent deposits in the retina. These findings demonstrate a strategy for the identification of new drug candidates and FDA-approved drugs for the treatment of monogenic and complex diseases.
\end{abstract}

\section{Introduction}

Decades of scientific advances have identified a large number of monogenic inherited (1-3) and complex genetic traits (4-7) that, along with environmental insults to organs and tissues with fully delineated specific genetic backgrounds (8-10), can drive disease progression in humans. In addition to identifying causes of these diseases, such as DNA sequence variants, it has been demonstrated that the activation of specific metabolic and signaling pathways can accelerate disease pathology $(11,12)$. Characterization of these pathways has also instigated ideas of potentially treating certain human conditions by either blocking or activating them (e.g., refs. $11,13)$, but in most cases, possible links between such pathways and human disease are unclear or unexplored. Moreover, some of these pathways at first glance appear unrelated. In recent years, new concepts of quantitative systems pharmacology have emerged that integrate systems biology and pharmacology (14-18). In the present genomic era, the process of integration has further advanced with massive sequencing data obtained from tissuespecific transcriptomes by next-generation sequencing that simultaneously provides quantitative identification of all transduction cascades $(19,20)$. This connection can improve our understanding of drug action from diverse classes of pharmaceuticals. Moreover, systems pharmacology promises to identify new uses of existing drugs for combined therapies.

The vertebrate retina is exceptionally suited for systems pharmacology because of its isolation from other organs and easy diagnostic access. Nurtured by a rich choroid coat situated between the photoreceptors/retinal pigment epithelium (RPE) and sclera $(21,22)$, the retina connects with the brain only

Conflict of interest: The authors have declared that no conflict of interest exists. Citation for this article: J Clin Invest. 2013;123(12):5119-5134. doi:10.1172/JCI69076. through the optic nerve but is accessible to various biochemical and electrophysiological analyses (23-25). Retinal degeneration and pathological changes can be monitored by three key noninvasive imaging techniques: scanning laser ophthalmoscopy (SLO) (26), optical coherence tomography (OCT) $(27,28)$, and two-photon microscopy (TPM) (29-33).

Stargardt disease, the most common juvenile form of retinal macular degeneration, is associated with more than 800 mutations in the ABCA4 gene (34). ABCA4 is an ATP-binding cassette transporter that shuttles all-trans-retinal (atRAL) from the internal membranes of retinal outer segment discs to the cytoplasm, where it is reduced to retinol by retinol dehydrogenases (RDHs) (35). Although the $\mathrm{Abca}^{-/-}$mouse model fails to mimic the human disease phenotype $(36,37)$, simultaneous removal of RDH8, the next enzyme in this retinoid pathway $(37,38)$, results in double-knockout $A b c a 4^{-/-} R d h 8^{-/-}$mice that exhibit many phenotypic changes found in patients with Stargardt disease. Here, the retinal pathology can be traced to the cytotoxic effect of atRAL (31). Exposure of Abca4 $4^{-/-} \mathrm{Rdh}^{-/-}$mice to bright light consistently increased atRAL levels in the retina, which induced rapid NADPH oxidase-mediated overproduction of intracellular ROS through a cascade implicating GPCR and PLC/IP $/ \mathrm{Ca}^{2+}$ signaling (39).

Despite delineating the genetic contributions that give rise to Stargardt disease, lack of understanding the precise pathways that drive this disease has resulted in inadequate therapeutics for this disorder. With the hypothesis that activation and/or inhibition of key GPCRs in the retina can affect disease progression, we used a systems pharmacological approach with $\mathrm{Abca4^{-/- }} R d b 8^{-/-}$mice that specifically targeted signal transduction by several different GPCRs and their interconnected mechanisms to identify novel therapeutic strategies for treating blinding retinal disorders such as Stargardt disease and age-related macular degeneration (AMD). 


\section{Table 1}

Expression of ARs, 5 -HT receptors, and ACs in the eye and retina of C57BL/6J mice, the eye of photoreceptor-degenerated $R h^{-/-}$mice, the retina of a human donor eye, and macular tissue from monkey (Macaca fasicularis) ${ }^{A}$

\begin{tabular}{|c|c|c|c|c|c|}
\hline Gene & $\begin{array}{l}\text { B6 mouse } \\
\text { eye }\end{array}$ & $\begin{array}{l}\text { B6 mouse } \\
\text { retina }\end{array}$ & $\begin{array}{c}\text { Rho- }^{-/} \text {mouse } \\
\text { eye }\end{array}$ & $\begin{array}{l}\text { Human } \\
\text { retina }\end{array}$ & $\begin{array}{l}\text { Monkey } \\
\text { macula }\end{array}$ \\
\hline \multicolumn{6}{|l|}{ ACs } \\
\hline Adcy1 & 17.53 & 37.50 & 9.72 & 68.60 & 81.26 \\
\hline Adcy2 & 14.41 & 21.70 & 22.96 & 5.78 & 11.42 \\
\hline Adcy3 & 5.59 & 5.09 & 1.57 & 5.70 & 21.21 \\
\hline Adcy 4 & 1.34 & 0.54 & 2.52 & 1.18 & 0.48 \\
\hline Adcy5 & 7.19 & 11.15 & 11.14 & 8.00 & 12.79 \\
\hline Adcy 6 & 17.68 & 49.98 & 11.41 & 9.99 & 7.25 \\
\hline Adcy 7 & 4.95 & 0.75 & 0.60 & 1.14 & 2.46 \\
\hline Adcy 8 & 2.13 & 3.80 & 2.76 & 3.73 & 8.94 \\
\hline Adcy 9 & 3.05 & 3.99 & 3.53 & 4.64 & ND \\
\hline Adcy10 & 0.02 & 0.03 & 0.01 & 0.01 & 0.33 \\
\hline \multicolumn{6}{|l|}{ ARs } \\
\hline Adra1a & 0.45 & 0.26 & 0.63 & 0.20 & 1.35 \\
\hline Adra1b & 1.60 & 1.62 & 2.35 & 1.12 & 6.61 \\
\hline Adra1d & 1.72 & 3.83 & 4.26 & 0.08 & 3.97 \\
\hline Adra2a & 4.60 & 9.91 & 6.14 & 0.25 & 0.67 \\
\hline$A d r a 2 b$ & 0.20 & 0.29 & 0.20 & 0.02 & 0.06 \\
\hline Adra2c & 8.96 & 7.17 & 19.28 & 2.38 & 4.30 \\
\hline \multicolumn{6}{|c|}{ 5-HT receptors } \\
\hline Htr1a & 0.17 & 0.28 & 0.13 & 0.15 & 0.16 \\
\hline Htr1b & 1.80 & 3.99 & 1.05 & 0.80 & 8.22 \\
\hline Htr1d & 1.49 & 2.58 & 1.91 & 0.00 & 0.07 \\
\hline Htr1f & 0.03 & 0.04 & 0.03 & 0.44 & 0.06 \\
\hline Htr2a & 0.67 & 0.72 & 0.68 & 1.30 & 0.67 \\
\hline$H t r 2 b$ & 0.50 & 0.43 & 0.30 & 0.39 & 0.86 \\
\hline Htr2C & 0.56 & 0.73 & 0.52 & 0.02 & 0.27 \\
\hline Htr4 & 0.02 & 0.01 & 0.01 & 0.43 & 0.05 \\
\hline$H t r 5 a$ & 0.43 & 0.59 & 0.48 & 0.00 & 2.17 \\
\hline Htr6 & 0.19 & 0.36 & 0.29 & 0.00 & 0.29 \\
\hline Htr7 & 0.31 & 0.31 & 0.41 & 0.01 & 0.59 \\
\hline \multicolumn{6}{|c|}{ Arrestins } \\
\hline Sag & 1220.54 & 1805.26 & 301.99 & 3562.22 & 2248.35 \\
\hline Arrb1 & 18.38 & 18.10 & 9.42 & 8.21 & 18.22 \\
\hline Arrb2 & 8.86 & 14.99 & 11.92 & 17.92 & 21.68 \\
\hline Arr3 & 50.73 & 128.48 & 32.62 & 270.16 & 680.86 \\
\hline \multicolumn{6}{|c|}{ GPCR kinases } \\
\hline Grk1 & 111.20 & 236.84 & 14.19 & 175.06 & 88.96 \\
\hline Adrbk1 & 28.70 & 36.88 & 36.15 & 27.29 & 32.71 \\
\hline Adrbk2 & 2.83 & 4.68 & 3.52 & 1.30 & 8.84 \\
\hline Grk4 & 0.86 & 0.90 & 0.42 & 5.31 & ND \\
\hline Grk5 & 3.08 & 2.97 & 2.57 & 2.99 & 5.06 \\
\hline Grk6 & 20.26 & 20.31 & 6.95 & 9.69 & 9.02 \\
\hline Grk7 & ND & ND & ND & 8.94 & 20.48 \\
\hline \multicolumn{6}{|c|}{ Photoreceptor genes } \\
\hline Abca4 & 59.00 & 140.14 & 15.85 & 267.71 & 129.68 \\
\hline Opn1sw & 117.79 & 187.21 & 72.95 & 25.42 & 151.31 \\
\hline Rho & 5853.00 & 11081.16 & 39.26 & 6386.12 & 8168.27 \\
\hline
\end{tabular}

AAnalyses were done as described in Methods. Greater expression in one compartment compared with another or from one species with another indicates their enrichment in that particular compartment. Values for photoreceptor genes Abca4, Opn1sw, and $R$ ho are shown for reference. ND, not determined because no gene homolog exists in the designated species. Preliminary results have been published $(47,83,88)$.

\section{Results}

Expression of GPCRs and GPCR signaling genes in buman and mouse retinas. Expression analysis of retinal GPCRs by immunocytochemistry was unreliable for reasons that include poor specificity and low affinity of antibodies (data not shown) as well as low GPCR expression. Therefore, we turned to quantitative transcriptome analysis of human and mouse retinas without specific cellular localization.

Overall, we conducted gene ontology analysis in Mus musculus and found 1,766 unique gene products categorized as having GPCR activity. The same mouse transcripts were also recognized in the retinal transcriptome from Homo sapiens. Of these 1,766, we found that 165 genes displayed expression of at least 1 fragment per kilobase of exon per million mapped reads (FPKM), equivalent to 1 transcript per cell, in C57BL/6J mouse eye or retina, with 6 genes below the 1-FPKM threshold in mouse eye, but above the 1-FPKM threshold in mouse retina (Supplemental Table 1; supplemental material available online with this article; doi:10.1172/JCI69076DS1). Expression of these 165 genes from human retina is also displayed as decreasing FPKM values starting from rhodopsin (RHO), the most highly expressed gene in mouse eye. The most highly expressed human GPCR was rhodopsin, followed by retinal $G$ protein receptor (RGR) and the cone pigments OPN1SW and OPN1MW.

We performed a more detailed analysis of adenylate cyclases (ACs), $\alpha 1$-adrenergic receptors ( $\alpha 1$-ARs), $\alpha 2$-ARs, and serotonin receptors (5-HTRs) to detect potential pathways that could be affected by a systems pharmacological approach. Reaction-quenching molecules, such as arrestins and GPCR kinases, were also investigated (Table 1). Expression values for these genes in mouse and human retinas highlighted those pathways that should be most susceptible to pharmacological treatment as well as those targets that would best translate from mouse models to human patients. For example, among isoforms of the $\alpha 1$-ARs and $\alpha 2$-ARs, the human retina expressed $\alpha 2 \mathrm{C}$-AR at the highest level (Table 1), and among 5-HTRs, 5-HT2AR had the highest expression. Data from real-time PCR analyses corroborated that the expression of these genes is readily detected in the mouse retina and/or RPE (unpublished observations of the authors).

To better understand GPCR localization in the eye and even the macula, we undertook more in-depth transcriptome studies. To potentially localize such transcripts to this photoreceptor layer, we conducted transcriptome studies of rhodopsin knockout mice (40), which exhibit no rod pigment expression and fail to form rod photoreceptors. We also performed transcriptome studies with macular tissue isolated from monkeys to learn whether these GPCRs localize there and potentially mediate the high-resolution vision disrupted in macular diseases such as Stargardt disease. Our results (Table 1) showed that robust expression of Adcy1 was present in all eye tissues, but it was attenuated in the rhodopsin knockout mouse, indic- 
A

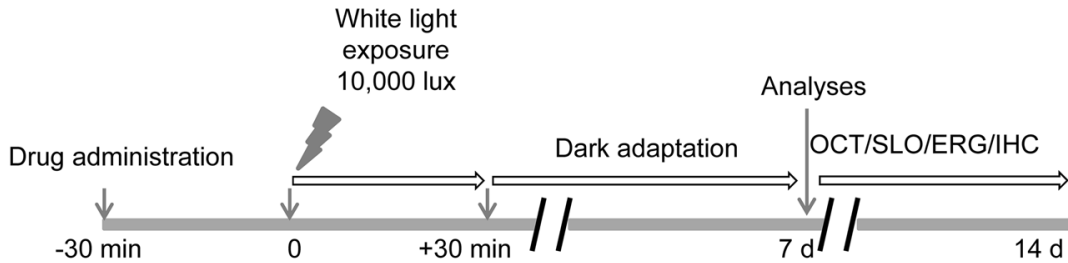

B

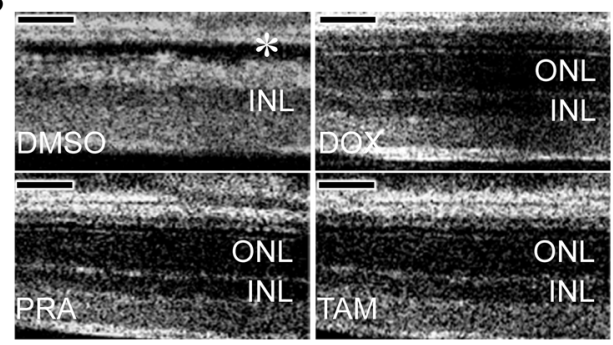

D

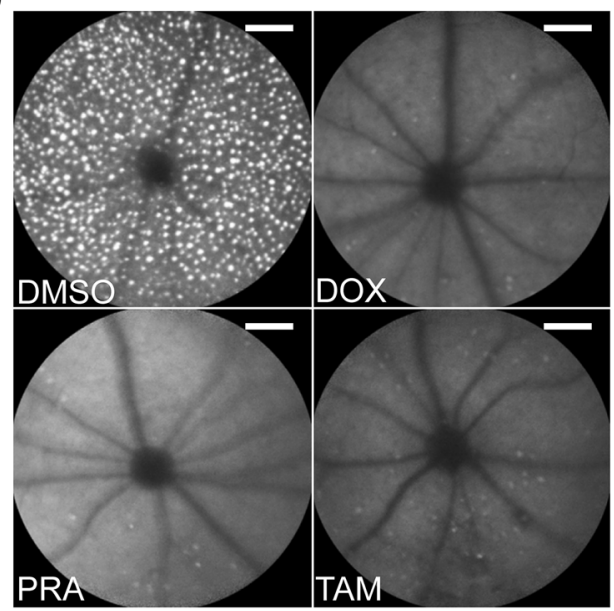

C

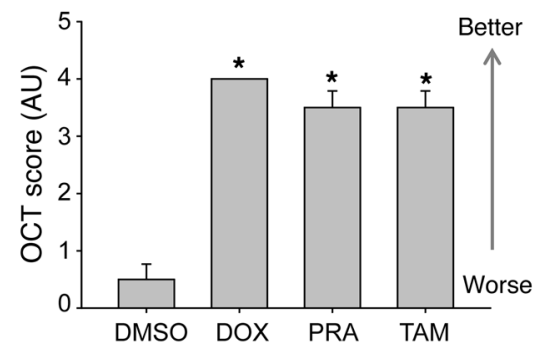

E

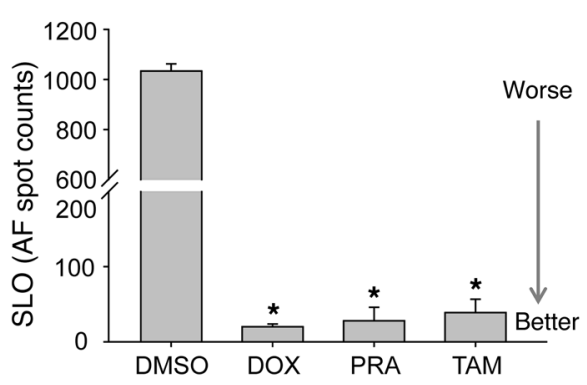

Figure 1

Antagonists of the $\alpha 1-\mathrm{AR}$, a Gq-coupled GPCR, protect $A b c a 4^{-/-} R d h 8^{-/-}$mouse retinas from bright lightinduced degeneration. (A) Schematic protocol for pharmacological treatment. All pharmacological com-

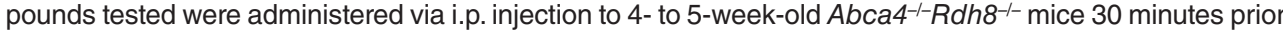
to white light exposure at 10,000 lux for 30 minutes. After light exposure, mice were kept in the dark for 7 to 14 days before functional and morphological examination by ERG, OCT, SLO, and IHC. (B) $\alpha 1$-AR antago-

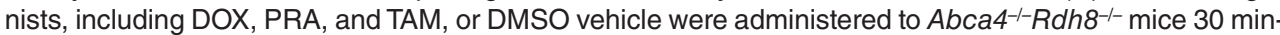
utes prior to white light exposure. OCT imaging was performed 7 days later to evaluate retinal morphology, and representative OCT images are shown. Asterisk indicates severely disrupted photoreceptor structures in vehicle-treated animals. ONL, outer nuclear layer; INL inner nuclear layer. (C) OCT scores from different treatment groups were subjected to statistical analysis (means $\pm \mathrm{SEM}$; ${ }^{*} P<0.01$ compared with DMSO control). (D) SLO imaging was performed 8 days after light exposure, and retinal autofluorescence images are shown. (E) Numbers of retinal autofluorescence (AF) spots were counted and subjected to statistical analysis (means \pm SEM; ${ }^{*} P<0.01$ compared with DMSO control). Scale bars: $50 \mu \mathrm{m}$.

5-week-old $A b c a 4^{-/-} R d b 8^{-/-}$mice shown previously to display severe light-induced retinopathy (38). These mice received DOX, PRA, or TAM 30 minutes prior to their exposure to white light at 10,000 lux for 30 minutes. We then kept the mice in the dark and evaluated the effects of each treatment 7-14 days later (Figure 1A). Noninvasive OCT imaging was performed after 7 days, revealing severely disrupted photoreceptor layers demonstrated by a diminished outer nuclear layer in DMSO vehicle-treated mice (31), whereas substantial protection of photoreceptor layer morphology was observed when these mice were pretreated with DOX, PRA, or TAM (Figure $1, \mathrm{~B}$ and $\mathrm{C}$ ). We also performed SLO imaging of retinal autofluorescence 8 days later to assess light-induced photoreceptor damage. We readily observed numerous autofluorescent spots in the retinas of Abca4 ${ }^{-/-} \mathrm{Rdh}^{-/-}$mice pretreated with DMSO control vehicle (Figure $1, \mathrm{D}$ and $\mathrm{E}$ ), a finding typical of light-induced photoreceptor damage (41). In contrast, pretreatment with DOX, PRA, or TAM significantly protected retinas from developing light-induced damage, as shown by a substantially reduced number of autofluorescent spots (Figure 1, $\mathrm{D}$ and $\mathrm{E}$ ), a finding consistent with the OCT imaging findings. These combined imaging results provide experimental evidence that pharmacological inhibition of $\alpha 1$-ARs can protect $\mathrm{Abca4}^{-/-}$ $R d h 8^{-/-}$mice against bright light-induced retinopathy.

Pharmacological compounds activating Gi-coupled $\alpha 2$-ARs protect against bright light-induced retinopative of its photoreceptor localization. We also noticed that Adcy1 expression was enriched in the monkey macula.

Pharmacological compounds targeting $\alpha 1$-ARs: inbibition of Gq-

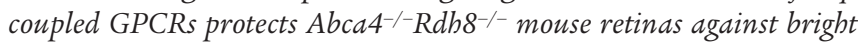
light-induced retinopathy. We evaluated pharmacological compounds antagonizing $\alpha 1$-ARs, a class of Gq-coupled GPCRs, for their effects on bright light-induced retinopathy according to our schematized protocol (Figure 1A). Three $\alpha 1$-AR antagonists including doxazosin (DOX), prazosin (PRA), and tamsulosin (TAM) (Supplemental Table 2) were independently tested in 4- to athy in $\mathrm{Abca4}^{-/-} \mathrm{Rdh}^{-/-}$mice. We also evaluated the Gi-coupled GPCR $\alpha 2-\mathrm{AR}$ as a possible target for treating photoreceptor degeneration. Agonists activating $\alpha 2$-ARs, including lofexidine (LOF), guanabenz (GUB), and guanfacine (GUF) (Supplemental Table 2), were each administered 30 minutes prior to exposure of 4- to 5-week-old $A b c a 4^{-/-} R d b 8^{-/-}$mice to bright light, and OCT images were taken 7 days later. Bright light-induced retinal morphological damage was largely prevented by pretreatment with GUB, GUF, or LOF as compared with the severe photoreceptor disruption manifested by DMSO-treated mice (Figure 2, A and B). Furthermore, we observed 
A

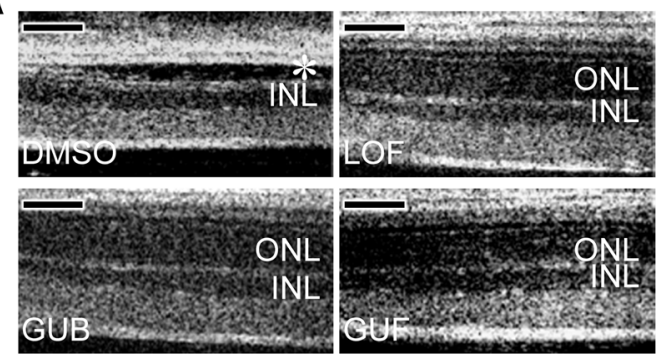

c

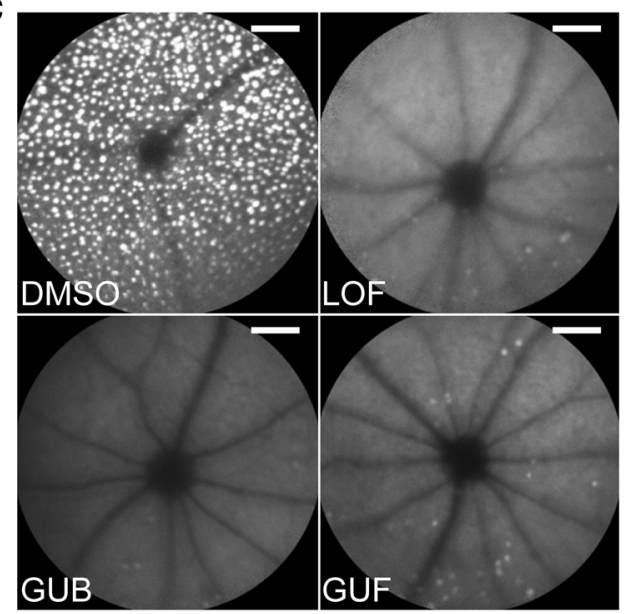

B

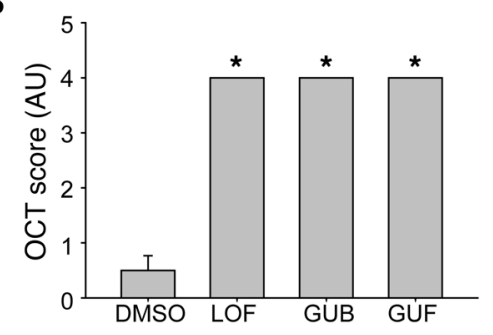

D

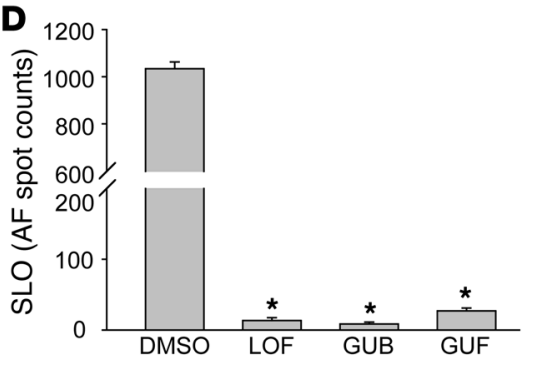

\section{Figure 2}

Agonists of the $\alpha 2-A R$, a Gi-coupled GPCR, protect $A b \mathrm{ca}^{-1-} \mathrm{Rdh} 8^{-/-}$mouse retinas from bright light-induced degeneration. The $\alpha 2-A R$ agonists LOF, GUB, and GUF were administered to 4- to 5-week-old $\mathrm{Abca4^{--- }} \mathrm{Rdh}^{-/-}$mice by i.p. injection 30 minutes prior to white light exposure at 10,000 lux for 30 minutes. DMSO was used as a vehicle control. (A) OCT imaging was performed to evaluate the effect of each treatment 7 days after light exposure. Asterisk indicates a markedly damaged photoreceptor structure in vehicle-treated control animals. (B) Statistical analysis of OCT scores (means \pm SEM; ${ }^{*} P<0.01$ compared to DMSO control). (C) SLO imaging was performed to detect autofluorescence 8 days after light exposure. Representative retinal autofluorescence images correlated positively with retinal damage. (D) SLO autofluorescence spot counts were further analyzed for statistical significance (means $\pm \mathrm{SEM}$; ${ }^{*} P<0.01$ compared to DMSO control). Retinal autofluorescence images correlated positively with retinal damage revealed by OCT imaging. Scale bars indicate $50 \mu \mathrm{m}$. a markedly decreased number of autofluorescent spots in mice pretreated with these compounds, in sharp contrast to the large number of bright autofluorescent spots seen in DMSO-pretreated mice (Figure 2, C and D). Clearly, retinal autofluorescence images correlated positively with retinal damage revealed by OCT imaging.

Pharmacological compounds inbibiting multiple Gs-coupled GPCRsprotect Abca $4^{-/-}$Rdh $8^{-/-}$mouse retinas from bright light-induced retinopatby. To further evaluate the therapeutic potential of compounds targeting other types of GPCRs, we also examined the effect of multiple antagonists of GPCRs coupled to Gs (Supplemental Table 2). We individually tested the antagonists that block activation of the 5-HT4 receptor RS 23579-190 (RS), the 5-HT6 receptors RO 04-6790 (RO) and SGS 518 oxalate (SGS), and the 5-HT7 receptors SB 269970 (SB) and LY 215840 (LY). We administered each compound to 4- to 5-week-old $A b c a 4^{-/-} R d h 8^{-/-}$mice 30 minutes before white light exposure at 10,000 lux for 30 minutes and performed OCT imaging 7 days later to evaluate retinal structural changes. In contrast to the dramatically damaged photoreceptor layer displayed by DMSOtreated, light-exposed $A b c a 4^{-/-} R d h 8^{-/-}$mice, we observed a marked preservation of the photoreceptor layer in $A b c a 4^{-/-} R d b 8^{-/-}$mice pretreated with compounds blocking the activation of Gs-coupled GPCRs such as the 5-HT4, 5-HT6, and 5-HT7 receptors (Figure 3, A and $\mathrm{B})$. The protective effects of the indicated compounds were further supported by significantly reduced formation of autofluorescent spots in $\mathrm{Abca4}^{-/-} \mathrm{Rdh8^{-/- }}$ mice treated with antagonists of these receptors (Figure 3, C and D). These results indicate that Gs-coupled GPCRs could also be further explored as plausible therapeutic targets for light-induced degenerative photoreceptor disorders.

Inbibition of AC prevents retinas from displaying marked light-induced degeneration. The above data demonstrate that multiple antagonists of Gs-coupled GPCRs or agonists of Gi-coupled GPCRs can protect retinas of $A b c a 4^{-/-} R d h 8^{-/-}$mice against bright lightinduced retinal degeneration (Supplemental Table 2). Given that $\mathrm{AC}$ is the central player mediating the intracellular function of both Gs- and Gi-coupled GPCRs, we tested the targeting of AC in our light-induced retinal degeneration mouse model. Thus, we administered the AC-selective inhibitor SQ 22536 (SQ) to Abca4 $4^{--}$ $R d h 8^{-/-}$mice 30 minutes before bright light exposure, followed by retinal structural examination by OCT imaging 7 days later. As shown in Figure 4, A and B, SQ treatment protected retinas from light-induced degeneration in a dose-dependent manner. Furthermore, SLO evaluation also demonstrated SQ's dosedependent prevention of autofluorescent spot formation, indicative of light-induced photoreceptor damage (Figure 4, C and D). These data are supportive of AC involvement in the pathogenesis of bright light-induced retinal degeneration. We performed an additional examination of the possible impact of AC inhibitor on phototransduction and found that amounts of 11-cis-retinal in the

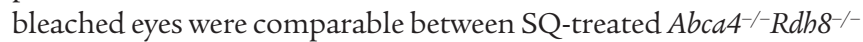
mice and vehicle-treated controls, whereas mice treated with the retinoid cycle inhibitor retinylamine showed markedly lower amounts of this visual chromophore. These data indicate that SQ treatment does not significantly change the visual cycle pigment recovery rate. Likewise, ERG responses from $A b c a 4^{-1-} R d b 8^{-/-}$mice were not affected by SQ administration under both scotopic and photopic conditions (unpublished observations of the authors).

Pharmacological interventions targeting Gq-, Gi-, Gs-coupled GPCRs, and AC preserve retinal morphology after exposure of $A b \mathrm{ca}^{-/-} \mathrm{Rdh8^{-/- }}$ mice to bright light. We performed immunohistochemical (IHC) examination to evaluate bright light-induced retinal morphological alterations in greater detail. We applied rhodopsin labeling of rod outer segments, peanut agglutinin (PNA) labeling of cone cell 
A

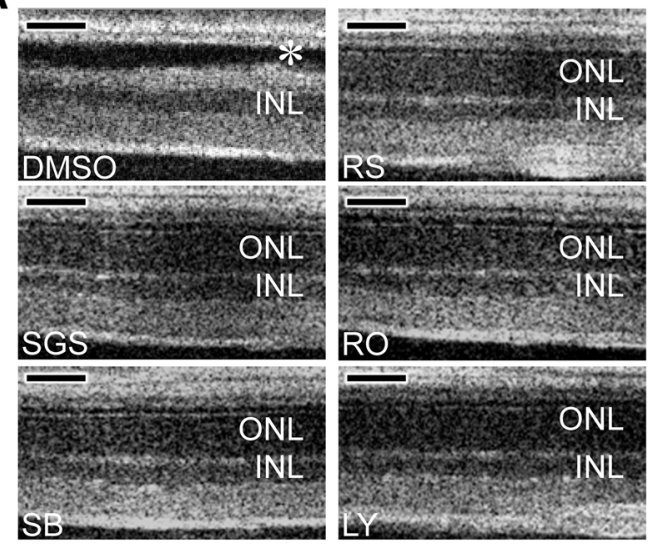

C

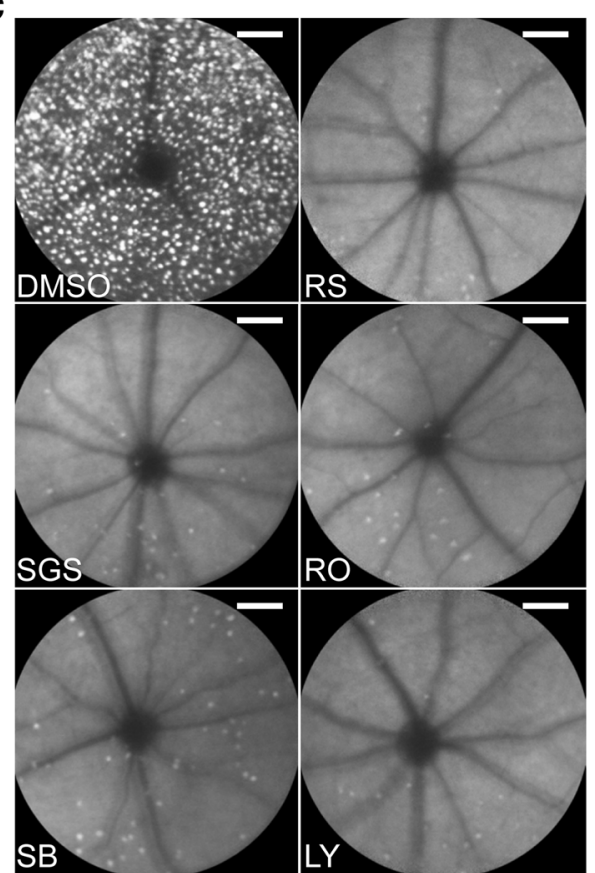

B

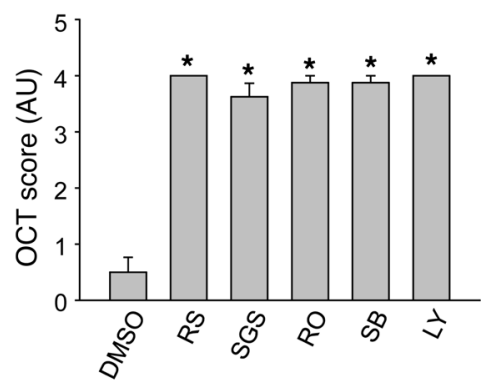

D

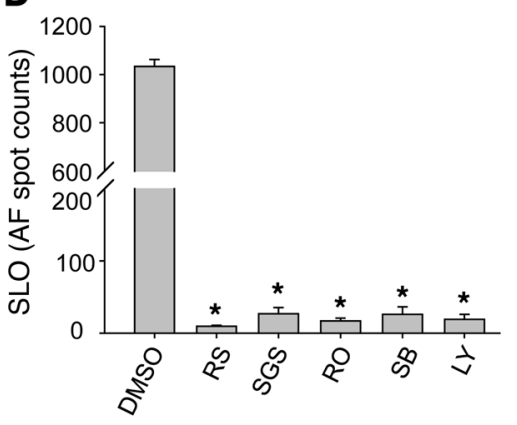

\section{Figure 3}

Multiple pharmacological antagonists of Gs-coupled GPCRs protect Abca4-/$R d h 8^{-/-}$mouse retinas from light-induced damage. RS, a 5-HT4R antagonist; SGS and $R O$, selective 5 -HT6R antagonists; SB, and LY, 5-HT7R antagonists; and the DMSO vehicle control were each administered to 4- to 5-week-old $A b c a 4^{-/-} R d h 8^{-/-}$mice by i.p. injection 30 minutes prior to white light exposure at 10,000 lux for 30 minutes. (A) The effect of each treatment was examined by OCT imaging 7 days after light exposure. Representative OCT images featured a disrupted photoreceptor structure only in the DMSO control, as indicated by the asterisk. (B) Statistically analyzed OCT scores (means \pm SEM; ${ }^{*} P<0.01$ compared with DMSO control). (C) SLO imaging was performed 8 days after light exposure. Representative retinal autofluorescence images show protection by all agents except DMSO. (D) Numbers of retinal autofluorescent spots were counted and statistically analyzed (means \pm SEM; ${ }^{*} P<0.01$ compared with DMSO control). Scale bars: $50 \mu \mathrm{m}$. matrix sheaths in the retina, and DAPI staining of cellular nuclei to retinal sections collected from $A b c a 4^{-/-} R d h 8^{-/-}$mice. As shown

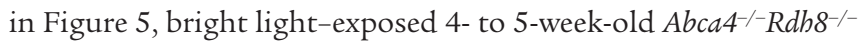
mice treated with DMSO vehicle displayed severely damaged photoreceptor structures manifested by only a residual expression of rhodopsin and PNA, together with a markedly decreased thickness of the DAPI-stained photoreceptor outer nuclear layer. In contrast, photoreceptor morphology was substantially better preserved, as indicated by abundant, well-organized rhodopsin and PNA expression as well as by a well-maintained DAPI-stained outer nuclear layer in $\mathrm{Abca4^{-/- }} R \mathrm{dh} 8^{-/-}$mice treated with pharmacological compounds targeting various GPCRs, including antagonists of Gq-coupled $\alpha 1$-ARs (DOX, PRA, and TAM), agonists of Gi-coupled $\alpha 2$-ARs (LOF and GUB), antagonists against either multiple Gs-coupled GPCRs such as 5-HT4R (RS), 5-HT6R (RO and SGS), and 5-HT7R (LY and SB), or AC (SQ).

Contribution of Gi and $\mathrm{Gq}$ pathways to light-induced retinal pathogenesis in the Abca4 $4^{-/}$Rdh8 $8^{-/-}$mouse model. To further elucidate the impact of Gi and Gq GPCR pathways on the pathogenesis of bright light- induced degeneration in $A b c a 4^{-/-} R d h 8^{-/-}$mice, we tested both the additive effects of Gi pathway activation and Gq pathway inhibition and the opposing effects of treatment with both idazoxan (IDA) (42), an $\alpha 2$-AR antagonist, and GUB, an $\alpha 2$-AR agonist. Both GUB (an activator of the Gi pathway) (Figure 6A) and DOX (an antagonist of the Gq pathway) (Figure $6 \mathrm{~B}$ ) protected the retinas of $A b c a 4^{-/-} \mathrm{Rdh}^{-/-}$mice from developing bright light-induced degeneration in a dose-dependent fashion. To further evaluate these positive effects, we first determined the half-maximal protective dose of GUB to be $0.3 \mathrm{mg} / \mathrm{kg}$ (Figure $6 \mathrm{~A}$ ) and that of DOX to be $0.4 \mathrm{mg} / \mathrm{kg}$ (Figure 6B). Then we found that treating mice simultaneously with half-maximal effective doses of GUB and DOX completely protected retinas from bright light-induced degeneration (Figure $6 \mathrm{C})$, indicating that simultaneous activation of Gi and inhibition of $\mathrm{Gq}$ pathways achieve retinal protection in an additive manner. Additionally, we verified that treating the mice first with the $\alpha 2-\mathrm{AR}$ antagonist IDA, followed by treatment with GUB, totally abolished the protective action of a fully effective dosage of the $\alpha 2$-AR agonist GUB. In distinct contrast to mice treated with GUB alone, retinas 
A

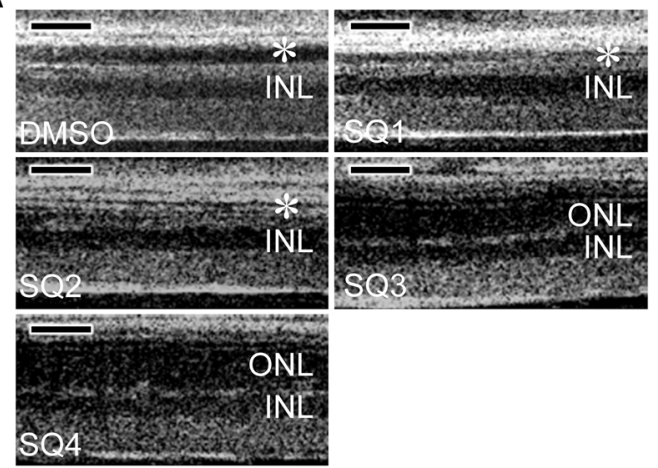

C

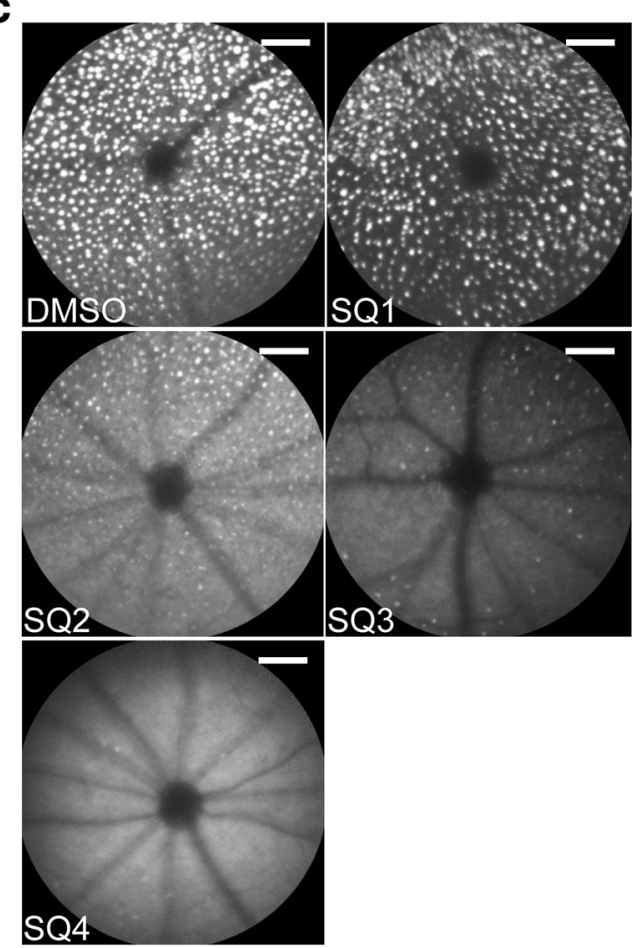

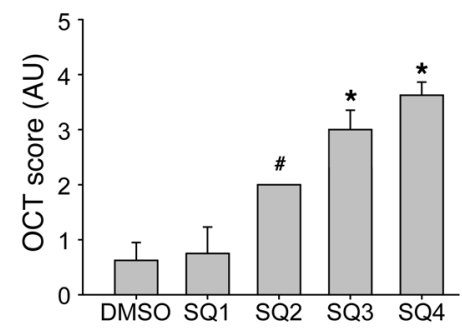

D

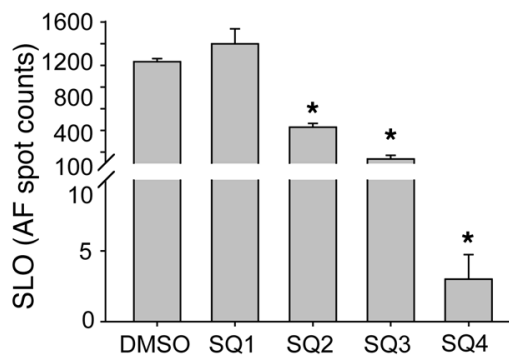

\section{Figure 4}

The selective AC inhibitor SQ protects $A b c a 4^{-/-} R d h 8^{-/-}$mouse retinas against bright light-induced damage in a dosedependent fashion. Increasing doses of $\mathrm{SQ}$ were administered to 4- to 5-weekold $A b c a 4^{--} R d h 8^{-/-}$mice by i.p. injection 30 minutes prior to white light exposure at 10,000 lux for 30 minutes. Doses were: SQ1: $0.083 \mathrm{mg} / \mathrm{kg}$; SQ2: $0.125 \mathrm{mg} / \mathrm{kg}$; SQ3: $0.25 \mathrm{mg} / \mathrm{kg}$; and SQ4: $0.5 \mathrm{mg} / \mathrm{kg}$. (A) The effect of SQ pretreatment was assessed by OCT imaging 7 days after light exposure. OCT images indicate damaged photoreceptor structures in DMSO-treated and a dose-dependent preservation of photoreceptor morphology in SQ-treated mice (asterisks identify damaged ONL). (B) Statistical analysis of OCT scores (means \pm SEM; ${ }^{\#} P<0.05$ and ${ }^{\star} P<0.01$ compared with DMSO control). (C) Retinal autofluorescence was examined by SLO imaging 8 days after light exposure. Representative retinal autofluorescent images reveal numbers and densities of bright spots that correlated with retinal damage. (D) Statistical analysis of the numbers of SLO autofluorescence spots (means \pm SEM; ${ }^{*} P<0.01$ compared with DMSO control). Scale bars: $50 \mu \mathrm{m}$. of mice pretreated with IDA and then with GUB were dramatically damaged. Moreover, this damage was more evident with an increased dose of IDA (Figure 6D). This last result further confirms the positive impact of activating the Gi pathway on retinal protection from bright light-induced degeneration.

Pharmacological interventions targeting Gq-, Gi-, Gs-coupled GPCRs, and $A C$ preserve retinal function. The effects of pretreatment with selected compounds affecting Gq-, Gi-, Gs-coupled GPCRs, and AC were also examined by scotopic and photopic ERG analyses performed 2 weeks after bright light exposure (Figure 7). Bright light exposure at 10,000 lux for 30 minutes nearly abolished the scotopic ERG response in $A b c a 4^{-/-} R d b 8^{-/-}$mice pretreated with DMSO vehicle (Figure 7, A and B). In marked contrast, we achieved substantial preservation of this response by pretreatments individually targeting multiple GPCRs. These included DOX, an antagonist of Gq-coupled $\alpha 1$-AR; RS, an antagonist of the Gs-coupled 5-HT4 receptor; RO, an antagonist of the Gs-coupled 5-HT6 receptor; LY, an antagonist of the 5-HT7 receptor; LOF, an agonist of the Gi-coupled $\alpha 2$-AR; and SQ, an inhibitor of AC. These data indicate that pharmacological treatment targeting these GPCRs also protects against light-induced retinal degeneration. A complete list of therapeutics is provided in Supplemental Table 2.

Pharmacological interventions targeting GPCRs and AC prevent lightinduced degeneration in WT mice. To investigate whether compounds that showed a protective effect against retinal degeneration in Abca4 $4^{-/} R d b 8^{-/-}$mice could also prevent retinal degeneration in WT mice, DOX ( $\alpha 1$-AR agonist), GUB ( $\alpha 2$-AR agonist), and the AC inhibitor SQ were further tested for their effect on light-induced retinal degeneration in $\mathrm{BALB} / \mathrm{c}$ mice. These drugs were administered to 4-week-old $\mathrm{BALB} / \mathrm{c}$ mice by i.p. injection 30 minutes before white light exposure at 10,000 lux for 1 hour. We assessed retinal morphology 7 days after light exposure by OCT imaging and histological examination. As shown in Figure 8, severe retinal degeneration was observed in the mice treated with DMSO vehicle, whereas retinal morphology of the drug-treated mice was maintained, and no obvious signs of retinal degeneration were observed. Moreover, SLO examination revealed an infiltration of microglia and macrophages into the subretinal space, as shown by 


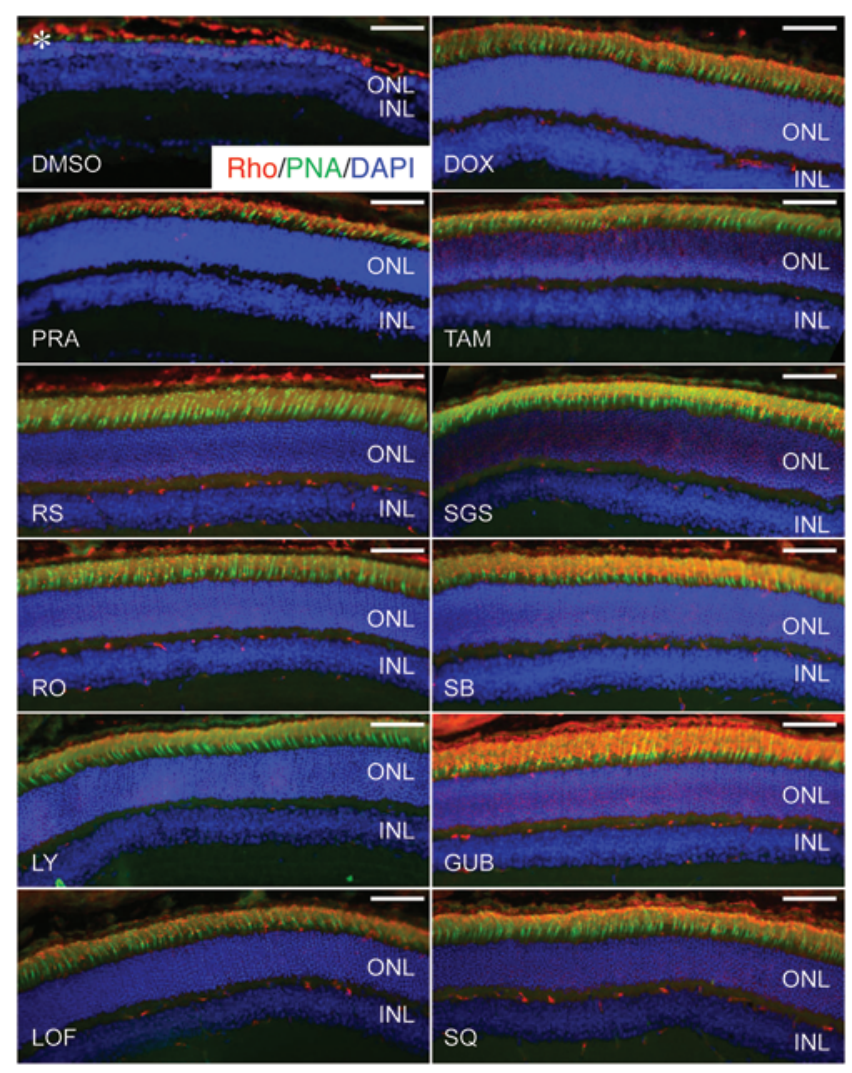

an increased number of autofluorescent spots in DMSO-treated and light-exposed mice, that was prevented in mice treated with DOX, GUB, or SQ. These results indicate that pharmacological interventions targeting GPCRs and AC could also prevent lightinduced degeneration in WT mice.

\section{Figure 5}

IHC examination shows protective effects on retinal morphology exerted by therapeutics targeting Gq-, Gs-, and Gi-coupled GPCRs

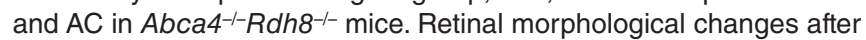
various indicated pretreatments were further evaluated by photoreceptor IHC examination (asterisk identifies severely reduced photoreceptor outer and inner segments manifested by only residual staining of rhodopsin and PNA in DMSO-treated control mice). Rho staining (red); PNA staining of cone extracellular matrix (green); DAPI staining of nuclei (blue). Scale bars: $50 \mu \mathrm{m}$.

Pharmacological interventions targeting GPCRs and AC inbibit ROS generation in mice after light exposure. The production of ROS is closely associated with photoreceptor cell death in $\mathrm{Abca}^{-/-} \mathrm{R} d \mathrm{~b}^{-{ }^{--}}$mice (39). Thus, we also examined compounds showing the protective effect against retinal degeneration in $A b c a 4^{-/-} R d b 8^{-/-}$mice for their ability to modulate ROS generation in $A b c a 4^{-/-} R d h 8^{-/-}$mice after light exposure. Dark-adapted 4- to -5-week-old Abca4-/-Rdh8 ${ }^{-/-}$ mice were i.p. injected with the fluorescent ROS probe DHE, together with DOX ( $\alpha 1$-AR agonist), GUB ( $\alpha 2$-AR agonist), or SQ (AC inhibitor) 30 minutes before light exposure at 10,000 lux for 1 hour. Figure 9 shows that among all the mice examined, the strongest ROS signals were detected in photoreceptor nuclei in DMSO-treated mice. Pharmacological interventions targeting GPCRs and AC were able to reduce ROS generation in $\mathrm{Abca4^{-- }}$ $R d h 8^{-1-}$ mice after light exposure. These results indicate that ROS generation is one of the common downstream pathways potentially mediating the effects of aberrant GPCR/AC signaling in light-induced retinal degeneration in $\mathrm{Abca4^{-/- }} \mathrm{Rdh}^{-/-}$mice.

Penetration of DOX, GUB, and SQ into mouse eyes. Though drugs administered into the systemic circulation are distributed throughout the body, they can also achieve different concentrations in various organs and tissues depending on the rates of vascular perfusion and the drugs' molecular properties, such as their lipid solubility, $\mathrm{pKa}$, and their ability to bind to carrier proteins. Moreover, drugs

\section{Figure 6}

Contributions of $\mathrm{Gi}$ and $\mathrm{Gq}$ pathways to lightinduced retinal pathogenesis. (A) GUB, a Gi pathway activator, protected $A b c a 4^{-/-} \mathrm{Rdh}^{-/-}$ mouse retinas from bright light-induced degeneration in a dose-dependent fashion with a halfmaximal effective dose of $0.3 \mathrm{mg} / \mathrm{kg} . n \geq 5$ for each data point. (B) The Gq pathway inhibitor DOX also protected mouse retinas from bright light-induced degeneration in a dose-dependent fashion with a half-maximal effective dose of $0.4 \mathrm{mg} / \mathrm{kg}$. $n \geq 5$ for each data point. (C) The combination of GUB at $0.3 \mathrm{mg} / \mathrm{kg} \mathrm{BW}$ and DOX at $0.4 \mathrm{mg} / \mathrm{kg} B W$ protected the retina in at least an additive manner; $n \geq 5$. (D) IDA at $2.5 \mathrm{mg} / \mathrm{kg}$ BW and $5 \mathrm{mg} / \mathrm{kg} \mathrm{BW}$ counteracted the protective action of GUB at $2 \mathrm{mg} / \mathrm{kg}$ BW on bright light-induced retinal degeneration; $n \geq 5$.
A
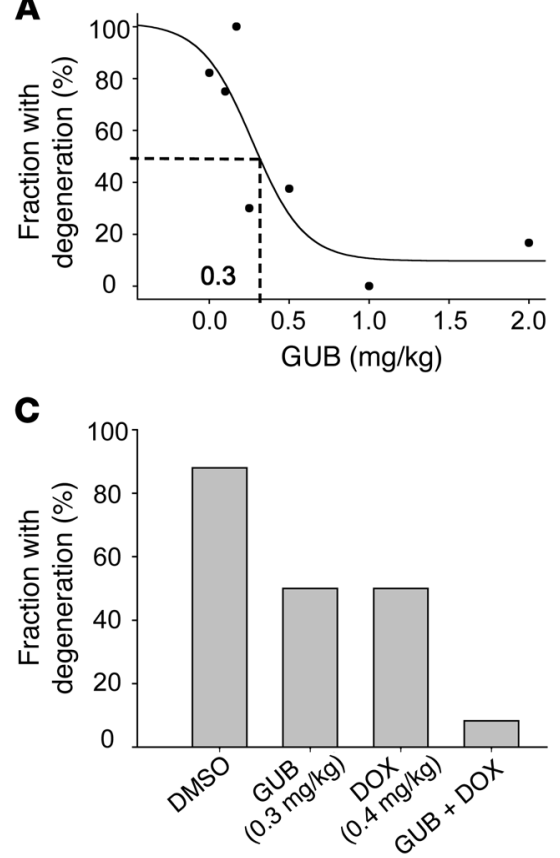

B

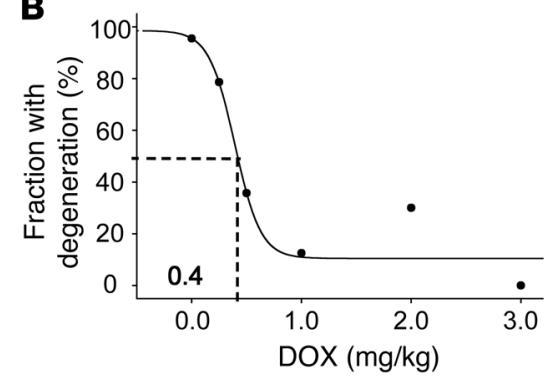

D

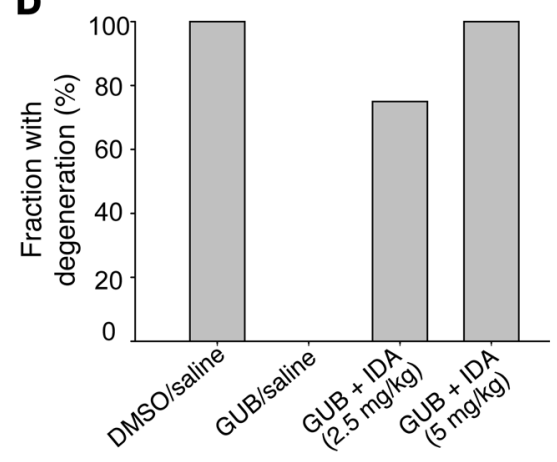



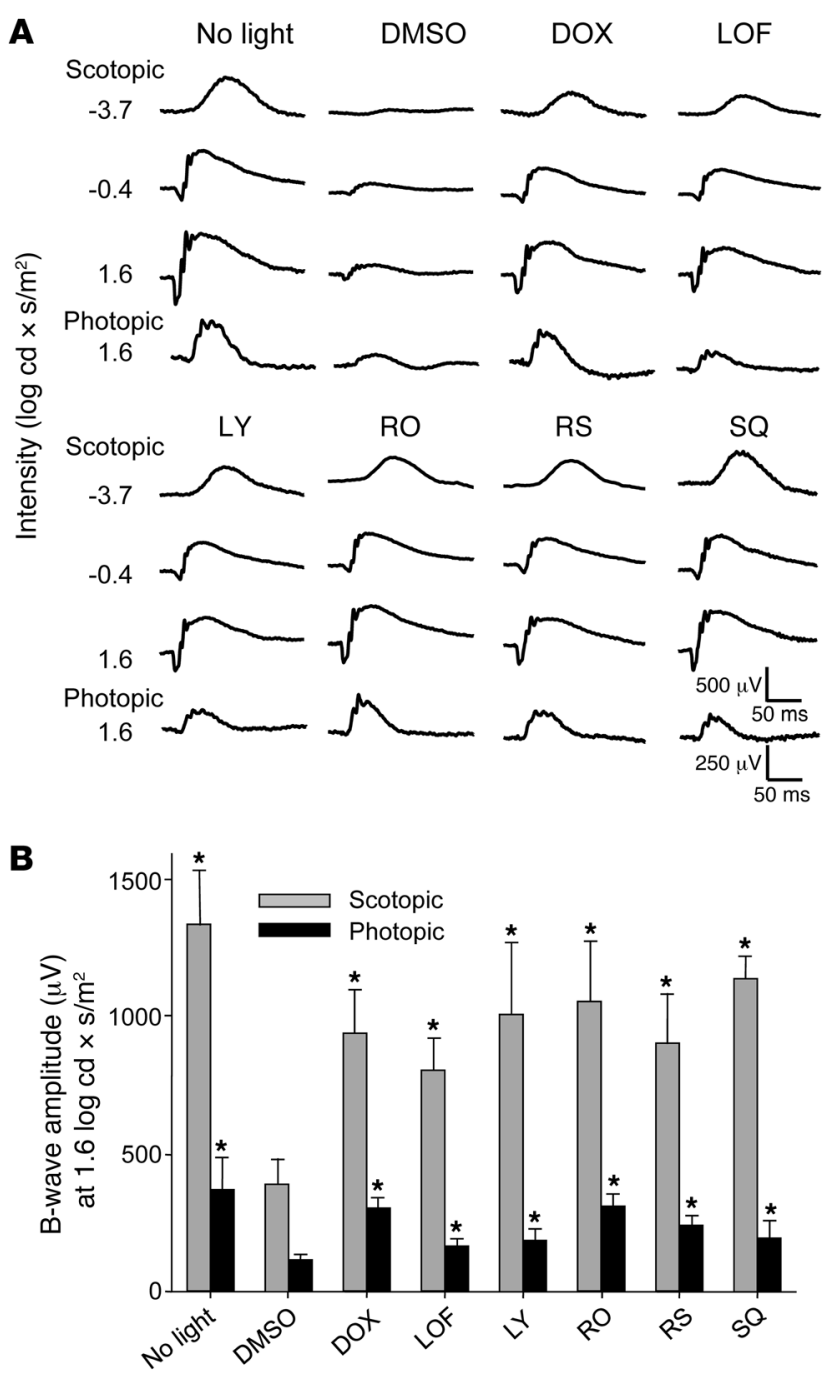

can also be cleared from target organs and tissues at different rates. To investigate whether DOX, GUB, and SQ can penetrate and persist in the eye, we quantified the amounts of these drugs present in the C57BL/6J (WT) whole-mouse eye globes within 2 hours after drug injection and compared these levels to those in serum. Liquid chromatography-mass spectrometry-based (LC-MS-based) analyses and quantification revealed the presence of tested compounds at levels in the low picomolar range in eye tissue (Table 2 and Figure 10). Given that the total volume of a mouse eye is about $0.1 \mathrm{ml}(43)$, the amounts of examined compounds were comparable to levels found in $0.1 \mathrm{ml}$ of serum samples for GUB and 5 times lower for both DOX and SQ. However, the retinal cell layer with an area of $15.6 \mathrm{~mm}^{2}$ (43) constitutes only a small fraction of the eye that likely absorbs most of these drugs entering from the blood. Thus, considering that the mice were intracardially perfused with PBS prior to harvesting the eyes, the amounts of DOX, GUB, and SQ confirmed their availability to eye tissue.

Pharmacological intervention targeting Gq-and Gi-coupled GPCRs prevents the formation of large fluorescent deposits in the RPE. The above results demonstrate a protective effect on photoreceptor morphology and function by pretreatment with pharmacological compounds targeting Gq-, Gi-, and Gs-coupled GPCRs. To investigate their impact on the RPE, we treated 4- to 5-week-old

\section{Figure 7}

Therapeutics targeting Gq-, Gs-, and Gi-coupled GPCRs and AC pre-

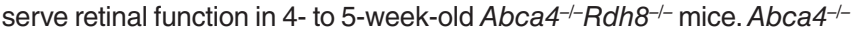
$R d h 8^{-1-}$ mice were exposed to 10,000 lux light for 30 minutes after pretreatment with the pharmacological agents DOX (10 mg/kg BW), LOF (2 mg/kg BW), LY (10 mg/kg BW), RO (30 mg/kg BW), RS (20 mg/kg $\mathrm{BW})$, and $\mathrm{SQ}(0.5 \mathrm{mg} / \mathrm{kg} \mathrm{BW})$. ERGs were recorded to evaluate the effects of these agents on retinal function 2 weeks after light exposure. (A) ERG responses were compared between mice not exposed to intense light (No light), vehicle only (DMSO), and tested agents under both scotopic and photopic conditions. Amplitudes of B waves at $1.6 \mathrm{log}$ $\mathrm{cd} \times \mathrm{s} / \mathrm{m}^{2}$ under scotopic and photopic conditions are shown (B). Tested compounds showed significant protective effects when compared with DMSO-treated mice, which displayed significantly impaired retinal function as indicated by decreased ERG amplitudes. ${ }^{\star} P<0.05$ compared with DMSO control. Bars indicate SDs. $n=4-6$ eyes per group.

albino Abca4 ${ }^{-/-} \mathrm{R} \mathrm{h} 8^{-/-}$mice with GUF, GUB, DOX, PRA, or TAM 30 minutes before bright light exposure. We assessed changes in RPE morphology in freshly enucleated mouse eyes by TPM performed 10 days after treatment. Mice exposed to bright light but not pretreated with the above compounds accumulated large, long wavelength-evoked fluorescent deposits in the RPE, which otherwise appeared structurally unaffected. Representative TPM images comparing the RPE from mice treated with PRA, GUB, and vehicle are shown in Figure 11A. Spectra of these granules, shown in Figure 11B, displayed broad maxima at 590 to $625 \mathrm{~nm}$, indicative of pyridinium bisretinoid, A2E, and related retinoids (44). These data indicate that treatment with compounds targeting Gq- and Gi-coupled GPCRs can prevent the RPE from accumulating potentially toxic long-wavelength fluorescent deposits.

\section{Discussion}

Stargardt disease is an inheritable juvenile macular dystrophy that leads to progressive vision loss and ultimate blindness. Most cases of Stargardt disease can be traced to mutations of the atRAL transporter ABCA4 $(34,45)$, and its pathology directly relates to the toxicity of atRAL (31). Others have postulated that condensation products of atRAL and further light exposure cause macular degeneration (46), but how atRAL actually triggers retinal degeneration is only partially understood. Toxicity induced by atRAL is mediated through a signaling cascade implicating GPCRs, PLC/ $\mathrm{IP}_{3} / \mathrm{Ca}^{2+}$ signaling, and NADPH oxidase (39). This discovery provided several new targets for halting progression of the disease in addition to free atRAL sequestration (31). It is also likely that atRAL plays some role in AMD, just as it does in Stargardt disease. Most plausible is that AMD results from an imbalance of several signaling pathways, such that attenuation or activation of these pathways could help preserve the retina. Genetic predisposition (47), diet, cigarette smoking, bright sunlight, cardiovascular disease, and hypertension are all risk factors for AMD (48). The age-related eye disease study (AREDS) demonstrated a substantial impact of taking dietary supplements containing high-dose antioxidants and zinc on halting disease progression and preserving visual acuity $(8,9)$.

In the retina, a nonredundant set of genes is primarily involved in processing the light signal and regenerating the light-sensitive chromophore $(35,49,50)$. But the retina, especially its rod and cone photoreceptors, is a highly labile tissue because of its high oxygen levels $(51,52)$, high levels of unsaturated lipids (53), high 

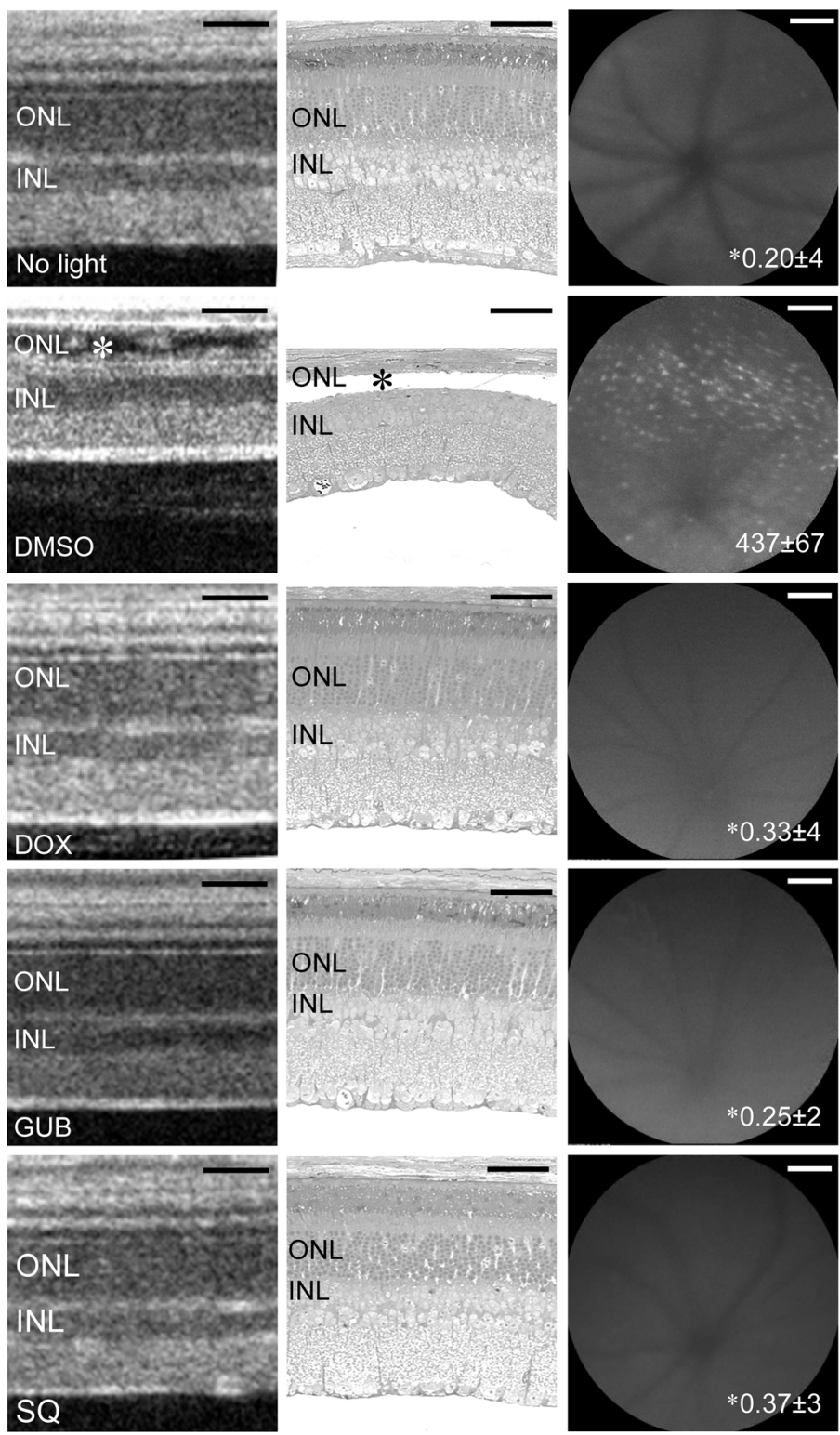

\section{Figure 8}

DOX, GUB, and SQ each prevent light-induced retinal degeneration in WT mice. The $\alpha 1$-AR antagonist DOX, the $\alpha 2$-AR agonist GUB, or the AC inhibitor SQ was given to 4-week-old WT BALB/C mice by i.p. injection 30 minutes prior to white light exposure at 10,000 lux for 1 hour. BALB/c mice were used to reduce absorption of light by the RPE pigment. Doses of each compound were as follows: DOX, $10 \mathrm{mg} / \mathrm{kg}$; GUB, $2.0 \mathrm{mg} / \mathrm{kg}$; and SQ, $0.5 \mathrm{mg} / \mathrm{kg}$. Effects of these compounds were evaluated by spectral domain optical coherence tomography (SD-OCT) imaging 7 days after light exposure. Representative images of SD-OCT $500 \mu \mathrm{m}$ away from the optic nerve head in the superior retina are shown in the left panels. Asterisks indicate damaged photoreceptor structures evident only in DMSO-treated control mice. Retinal cross-sectional images of plastic sections (middle panels) were obtained from areas similar to those used for the OCT images. Retinal autofluorescence also was examined by SLO 7 days after light exposure (right panels). Numbers (means \pm SEM) of bright spots are indicated at the right bottom of the SLO images. Scale bars: $50 \mu \mathrm{m}$.

GPCRs, activating Gi-coupled GPCRs, blocking Gs-coupled GPCRs, or directly inhibiting AC prevented retinal degeneration to different degrees (Figure 12). First, without establishing their precise localization in the retina, we tested the $\alpha 1$-AR antagonists, a group of the three highly homologous subtypes $\alpha 1 \mathrm{~A}-\mathrm{AR}, \alpha 1 \mathrm{~B}-\mathrm{AR}$, and $\alpha 1 \mathrm{D}-\mathrm{AR}$, the latter of which is most highly expressed in mouse retina (Table 1). High doses of long-lasting DOX, a selective $\alpha 1$-AR blocker, effectively protected retinal tissue from light-induced damage (Figure 1 and Supplemental Table 2), supporting a previous study that identified Gq-coupled GPCR blockage as a target (ref. 39 and Figure 12). This compound, which confers positive effects on lipid profiles, is used to treat hypertensive patients and also those with urinary retention due to benign prostatic hyperplasia (63-65). Other similar antagonists, such as short-acting PRA and the antiprostatic hyperplasia drug TAM, were consistently effective, albeit with lower efficacy $(66,67)$. The presence of $\alpha 1$-ARs in rabbit retina has been reported, although their exact compartmental location remains to be established (68). Given the role of Gq-coupled GPCRs in light-induced retinopathy (39) and that $\alpha 1-\mathrm{AR}$ is an important member of this class of GPCRs, further efforts should be made to identify the location of $\alpha 1$-ARs and to clarify their physiological significance and therapeutic targeting.

The $\alpha 2$-ARs associated with the Gi-inhibitory heterotrimeric $G$ protein consist of three highly homologous subtypes that include $\alpha 2-\mathrm{AR}, \alpha 2 \mathrm{~B}-\mathrm{AR}$, and $\alpha 2 \mathrm{C}-\mathrm{AR}$. The $\alpha 2 \mathrm{C}$-ARs, previously localized to the soma and the inner segment of photoreceptors (69), are the most highly expressed receptors of this group in the human retina (Table 1). Activation of these receptors, accomplished by the blood pressure-lowering drugs GUB, GUF, and LOF at relatively low doses, exhibited the most consistent protective effects in all tested mice (Figure 2 and Supplemental Table 2). GUF did not induce ophthalmological deterioration, even after long-term treatment (70, 71), whereas LOF eye drops effectively increased the pupil diameter and lowered intraocular pressure (72). Interestingly, Allergan Inc. is currently conducting phase II clinical trials of the $\alpha 2 \mathrm{~A}-\mathrm{AR}$ agonist brimonidine tartrate for the treatment of AMD (ClinicalTrials.gov Identifier: NCT00658619), but results are not yet available.

Interestingly, even a low dose of a direct AC inhibitor such as SQ was highly protective against retinal degeneration in our experi- 

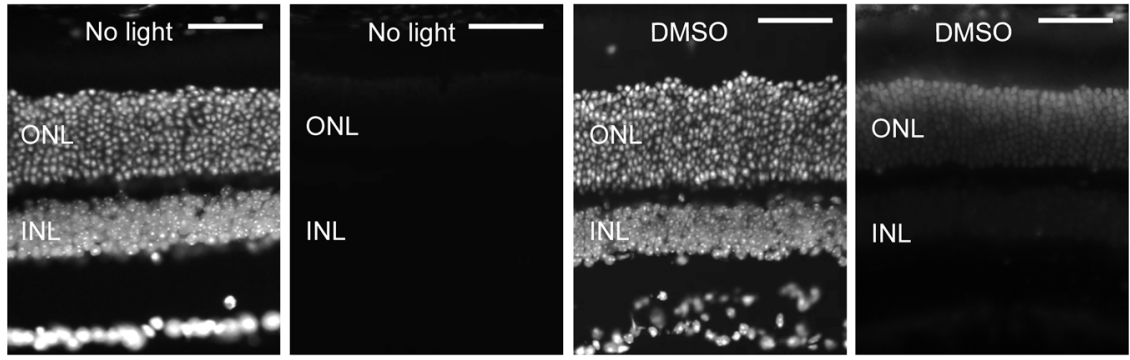

DOX
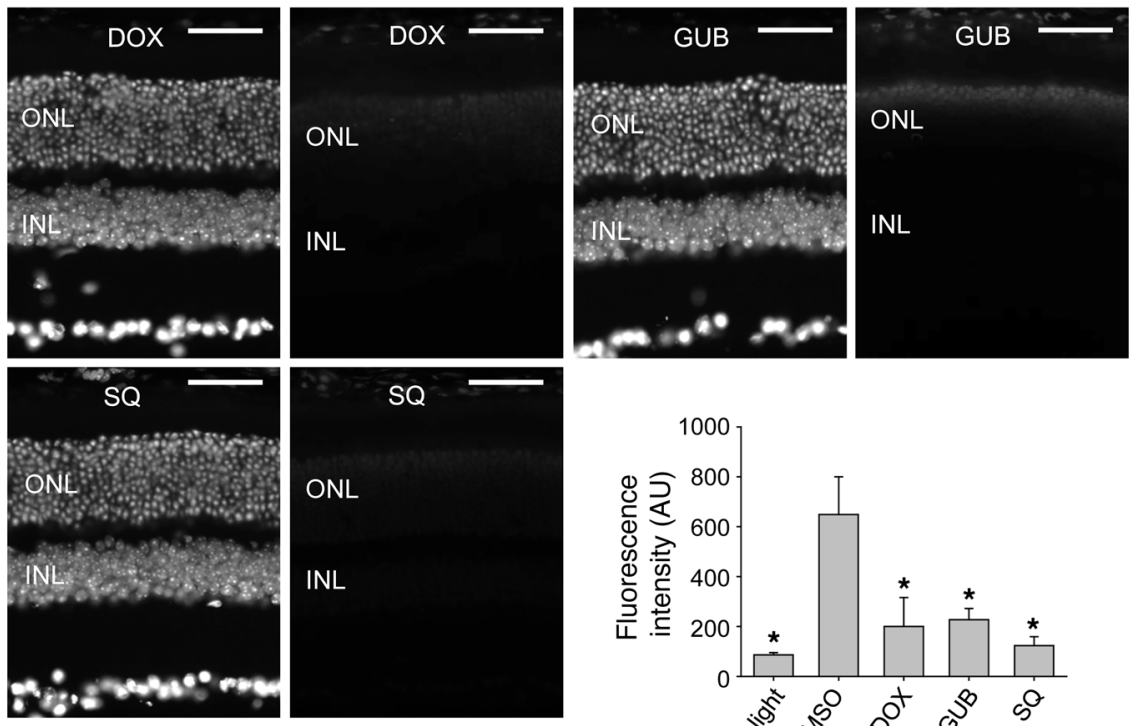

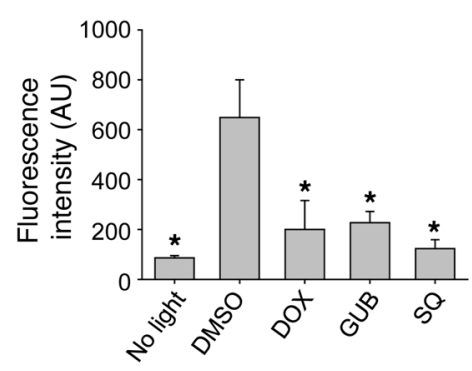

\section{Figure 9}

ROS generation in photoreceptors of $\mathrm{Abca4}^{-/-}$ $R d h 8^{-/-}$mice after bright light exposure is decreased by either DOX, GUB, or SQ pretreatment. Dark-adapted pigmented 4- to 5-weekold $A b c a 4^{-/-} \mathrm{Rdh}^{-/-}$mice were treated with the ROS probe DHE 1 hour prior to light exposure at 10,000 lux for 30 minutes. Either vehicle control (DMSO), DOX, GUB, or SQ were also administered by i.p. injection 30 minutes prior to light exposure. The dose of each compound was as follows: DOX, $10 \mathrm{mg} / \mathrm{kg}$; GUB, $2.0 \mathrm{mg} / \mathrm{kg}$;

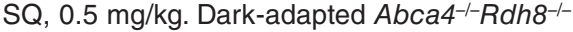
mice not exposed to light were included for DHE probe treatment as well (no light). Retinas were harvested 3 hours after illumination. ROS signals were obtained with the identical exposure setup under a fluorescence microscope (right panel of each image set). DAPI staining was performed as well to visualize cell nuclei and gross retinal structure (left panel of each image set). Recorded ROS fluorescence intensity in arbitrary units averaged from various areas was further analyzed and summarized for group comparisons (means \pm SEM). ${ }^{*} P<0.05$ compared with DMSO control. Scale bars: $50 \mu \mathrm{m}$. mental setup (Figure 4 and Supplemental Table 2). Ca ${ }^{2+}$-stimulated AC1 is the most highly expressed AC in the retina, and an initial immunocytochemical analysis of these enzymes in the retina has been reported (73). All AC isoforms are present in the inner plexiform layer, and both $\mathrm{Ca}^{2+}$-sensitive isoforms of $\mathrm{AC}$ (AC1 and $\mathrm{AC} 3$ ) and $\mathrm{Ca}^{2+}$-insensitive isoforms (AC2 and $\left.\mathrm{AC} 4\right)$ are expressed in ganglion cells. $\mathrm{Ca}^{2+}$-sensitive isoforms are more readily detected and expressed at higher levels in the outer retina, including photoreceptors and the outer plexiform layer.

Transgenic C. elegans expressing rhodopsin serves as a valid model for light-induced behavioral studies (74). DOX, GUB, or SQ was also found to be able to prevent atRAL-induced neurodegeneration in this C. elegans model (unpublished observations of the authors), further supporting the notion that atRAL-induced toxicity is mediated by GPCR pathways affected by these compounds.

Among the 5-HTRs we tested, the 5-HT2AR (Gq) antagonist nefazodone was highly protective (Supplemental Table 2), but high doses were required, and a liver damage side effect caused this drug to be withdrawn from the US market in 2004. Antagonists of Gs-coupled 5-HT4R (RS), 5-HT6R (RO and SGS), and 5-HT7R (SB and LY), which are used to induce relaxation and treat cognitive impairments such as amnesia, anxiety, and depression in patients, were effective in protecting mouse retina only at high doses (Supplemental Table 2), in which possible side effects could pose a problem. For example, transient but severe electrocardiographic changes and episodes of premature ventricular contractions were observed in patients when RS was administered at a dose that effectively blocked the 5-HT4R activity associated with periods of electrical-mechanical dissociation, leading to severe hypotension due to impaired cardiac function (75). Among these receptors, 5-HT4R is most highly expressed in the human retina (Table 1). mRNA expression from various subtypes of 5-HTRs in human retina has been documented, including 5-HT2, 5-HT3, 5-HT4, 5-HT5, and 5-HT7, with 5-HT2 expression being the most readily detected (76). In the mouse and rat, the presence of mRNAs for 5-HT1, 5-HT-2AR, 5-HT3, 5-HT6, and 5-HT7 was demonstrated (77), supporting the involvement of 5-HT1 and 5-HT2A (39) in retinal function under certain circumstances. This last finding also implies the possible therapeutic value of targeting other HTs such as 5-HT6 and 5-HT7. Alcon Inc. carried out phase II clinical trials for the treatment of atrophic/AMD with tandospirone, an eye drop formulation of a 5-HT1AR agonist used as a neuroprotective agent against excitotoxic neuronal damage, but results showed the treatment was ineffective, and

\section{Table 2}

Amounts of selected drugs found in the mouse serum and enucleated eyes 30 minutes after administration of a single dose

$\begin{array}{lcc}\text { Name }^{A} & \text { Serum }(\text { pmol } / \mathbf{1 0 0} \mu \mathrm{l}) \pm \text { SD } & \text { Eye }(\text { pmol/eye }) \pm \text { SD } \\ \text { DOX } & 46.5 \pm 12.8 & 11.0 \pm 1.7 \\ \text { GUB } & 7.0 \pm 1.8 & 13.1 \pm 4.7 \\ \text { SQ } & 19.3 \pm 5.5 & 5.6 \pm 2.2\end{array}$

ADOX, GUB, and SQ were detected and quantified in tissues of C57BL/J WT mice by LC-MS. All three drugs were found to penetrate and persist in the eye, with GUB reaching a higher concentration in the eye than in the serum. 
A

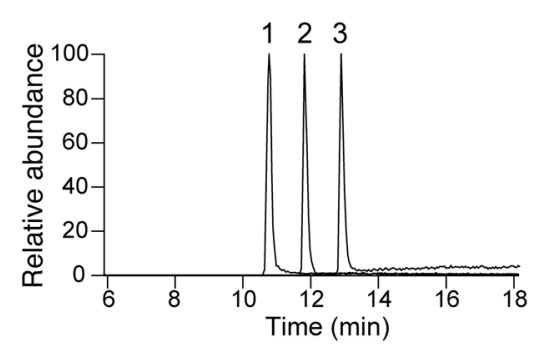

E

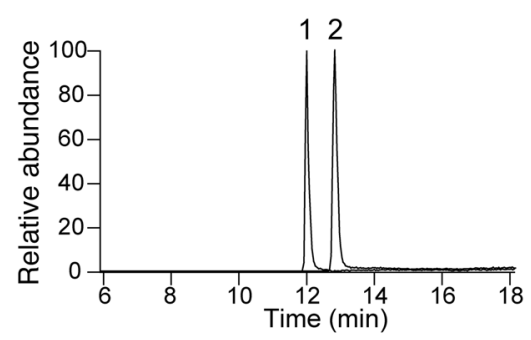

I

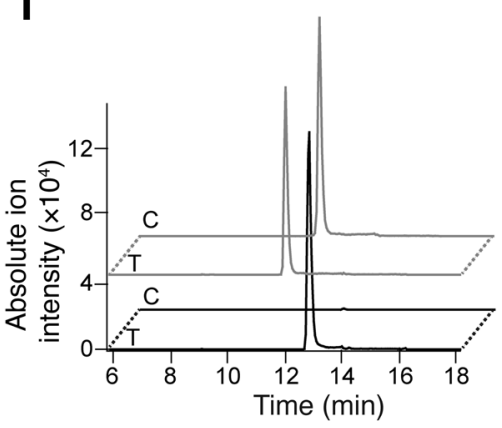

B

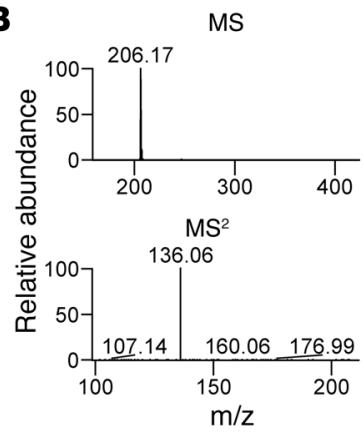

$\mathbf{F}$

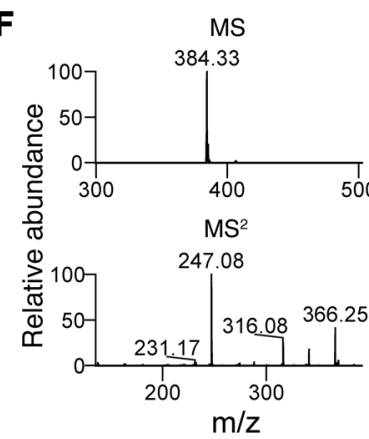

J

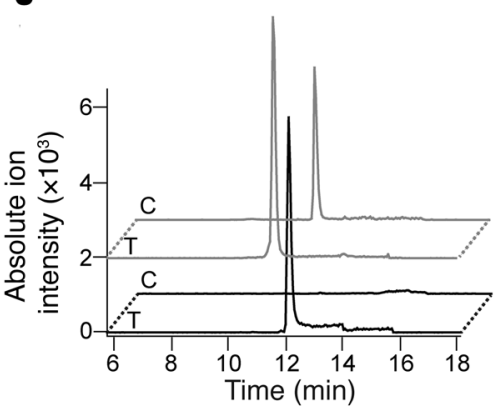

C

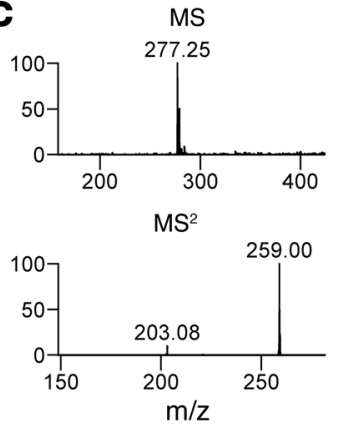

G

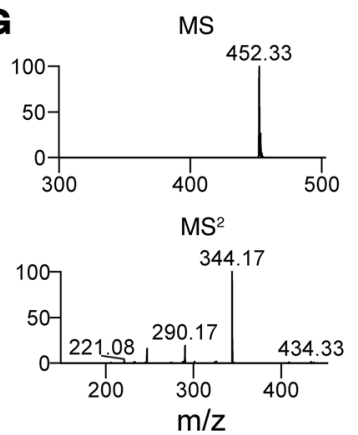

D
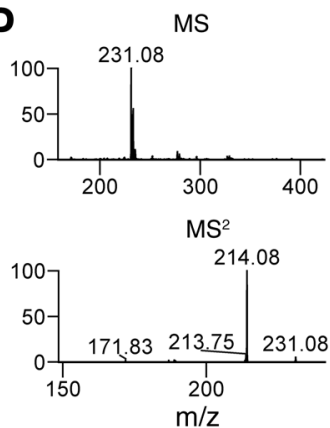

H

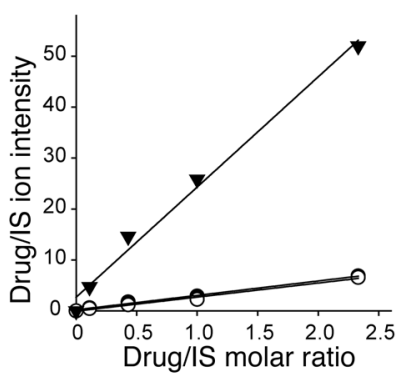

Figure 10

Detection and quantification of DOX, GUB, and SQ in mouse eye. (A) HPLC separation of SQ (peak 1), clenbuterol (IS) (peak 2), and GUB (peak 3). (B-D) MS and MS² patterns for SQ, clenbuterol, and GUB, respectively. Characteristic fragmentation profiles were used to design the selected reaction monitoring-based detection and quantification method. (E) Elution profile of PRA (IS) (peak 1) and DOX (peak 2). (F and G) MS and $\mathrm{MS}^{2}$ fragmentation pattern for PRA and DOX. (H) Relationship between ion intensities and molar ratio for drug/internal standard pairs (DOX/PRA [black triangles] and overlapping black and white circles for GUB/clenbuterol and SQ/clenbuterol, respectively), which were used for IS-based drug quantification. (I-K) Representative chromatograms of the eye extract indicating the presence of DOX, GUB, and SQ, respectively. Black chromatograms correspond to ion intensities of SRM transitions characteristic for the tested drugs. Gray lines represent ion intensities for the ISs. Letters " $\mathrm{T}$ " and "C" discriminate between samples obtained from drug-treated mice (T) and control, nontreated animals (C).

this study has been terminated (ClinicalTrials.gov). It also should be noted that we found this receptor to be very weakly expressed in human retina (Table 1 ).

Our results indicate that GPCR pathways provide distinct activation and inhibitory actions that could control photoreceptor cell survival. Thus, activation of the Gq pathway, accomplished by the $\alpha 1-\mathrm{AR}$, among others, can lead to photoreceptor cell death through the phospholipase C-mediated signaling pathway (79). Photoreceptor death can also result from aberrant functioning of Gs and Gi pathways that modulate the formation of cAMP (79). Gs, affected by the action of the 5-HT4, 6, and 7 receptors, activates this pathway, whereas $\mathrm{Gi}$, mediated by the $\alpha 2-\mathrm{AR}$, inhibits it. Therefore, the degenerative photoreceptor phenotypes in patho- logical states could be abrogated by pharmacological inhibition of either Gq or Gs pathways or by activation of the Gi pathway.

It is also worth noting that rhodopsin could be a central player in light-induced retinal degeneration (80) and that reduced amounts of rhodopsin protect the retina from light-induced degeneration $(81,82)$. Therefore, we examined the potential impact of the AC inhibitor SQ on chromophore regeneration, given that $\mathrm{AC}$ is the central player in our newly identified GPCR signaling implicated in light-induced retinopathy (Figure 12). When the effects of SQ treatment on ERG responses and 11-cis-retinal levels after bleaching were evaluated, no significant changes were observed (unpublished observations of the authors), indicating that the protection against retinal degeneration conferred by SQ treatment was 
A
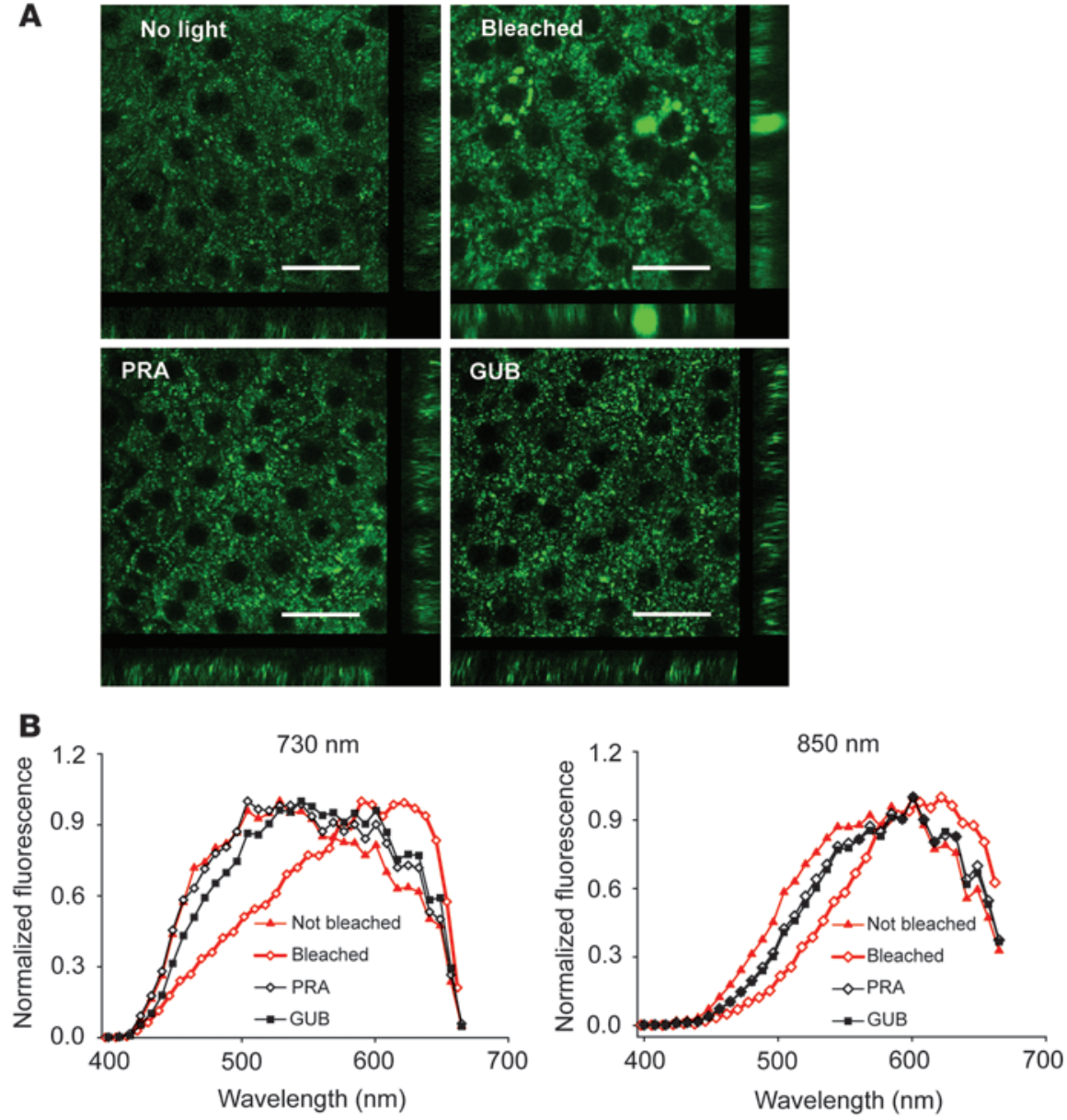

\section{Figure 11}

GPCR-targeted therapeutics prevent formation of large fluorescent granules in the RPE of 6- to 7-week-old Abca4 $4^{-/-}$Rdh $8^{-/-}$mice after exposure to bright light. (A) Representative TPM images of the RPE 10 days after exposure to bright light. Upper left panel, unexposed to light (No light) control; upper right panel, exposed to bright light (Bleached) and DMSO-treated control; lower left panel, pretreated with PRA; lower right panel, pretreated with GUB. Cross sections shown at the right edge and at the bottom of each en face RPE image reveal that fluorescent granules, most pronounced in the bleached DMSO-treated control, extend across the whole thickness of the RPE and into the outer retina-photoreceptor space. Scale bars: $25 \mu \mathrm{m}$. (B) Emission spectra after excitation with $730 \mathrm{~nm}$ light (left panel) and after excitation with $850 \mathrm{~nm}$ light (right panel). The spectra from light-exposed, DMSO-treated control are notably red-shifted for both excitation wavelengths. unlikely due to inhibition of visual pigment regeneration. Therefore, we were able to rule out the possibility of an acute effect of pharmacological compounds on phototransduction.

Together, the experimental results described here and in a previous publication (39) identify a series of intrinsically linked events, including the participation of GPCRs, $\mathrm{PLC} / \mathrm{IP}_{3} / \mathrm{Ca}^{2+}$ signaling, and NADPH oxidase-mediated ROS production, which are collectively responsible for the pathogenesis of retinal dystrophy in $A b c a 4^{-/-} R d h 8^{-/-}$mice, a model for rod/cone degeneration resembling features of human Stargardt disease. Our findings show that atRAL toxicity in bright light-induced retinal degeneration could be mediated through a signaling cascade implicating GPCRs, PLC/ $\mathrm{IP}_{3} / \mathrm{Ca}^{2+}$ signaling, and NADPH oxidase (39). Here, we report that, in addition to $\mathrm{Gq}$ signaling (Figure 1, new and confirmatory observations of our previous study), inhibition of the cAMP pathway also has a protective effect against retinal degeneration (Figures 2-4).

As presented here, the degenerative photoreceptor phenotypes were reversed by pharmacological inhibition of either Gq or Gs pathways or by activation of the Gi pathway (Figure 12). These proof-of-concept studies demonstrate interactions between interconnected and diverse pathways involved in the pathogenesis of retinal degeneration induced by strong light (39). Thus, systems pharmacology promises to greatly increase our knowledge of the mechanisms underlying the multiple actions of drugs and their cross-activation and inhibition of different retinal pathways involved in vision (Figure 12).

\section{Methods}

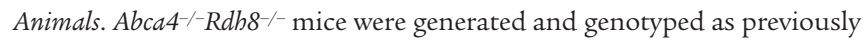
described (39), and mixed sexes were used for the present study when they reached 4-5 weeks of age. All mice were routinely maintained in a 12-hour light ( $\leq 10$ lux)/12-hour dark cyclic environment in the Animal Resource Center at the School of Medicine, Case Western Reserve University (CWRU). For bright light exposure experiments, $A b c a 4^{-/-} R d h 8^{-/-}$mouse pupils were dilated with $1 \%$ tropicamide prior to white light exposure at 10,000 lux (150 W spiral lamp; Commercial Electric) for 30 minutes. Alternatively, 4-week-old BALB/c mice (The Jackson Laboratory) were treated with the compounds indicated in Supplemental Table 2 via i.p. injection 30 minutes prior to 1 hour of white light exposure at 10,000 lux using a 150-W spiral lamp. Assessment of retinal structural and functional changes was performed 7 days after light exposure. Rhodopsin knockout mice ( $\left.R h o^{-/-}\right)$were obtained from Janis Lem (Tufts University, Medford, Massachusetts, USA) (40). Enucleated macaque (Macaca fascicularis) eyes in RNAlater (Invitrogen) from 4-year-old animals were obtained from Ricerca Biosciences. The retina was carefully dissected from an untreated eye requiring enucleation for a large ocular melanoma and immediately placed in RNAlater.

Transcriptome analyses of the eye and retina. Eyes from 4-week-old C57BL/6J mice (The Jackson Laboratory) were enucleated and immediately processed to isolate total RNA that was used to prepare cDNA libraries for sequencing with the Illumina platform of RNA sequencing instruments $(47,83)$. Three biological replicates were made for both whole-eye and retinal tissue to generate transcriptome data used to determine the FPKM for normalization and differential expression analyses. The retina was carefully dissected from 


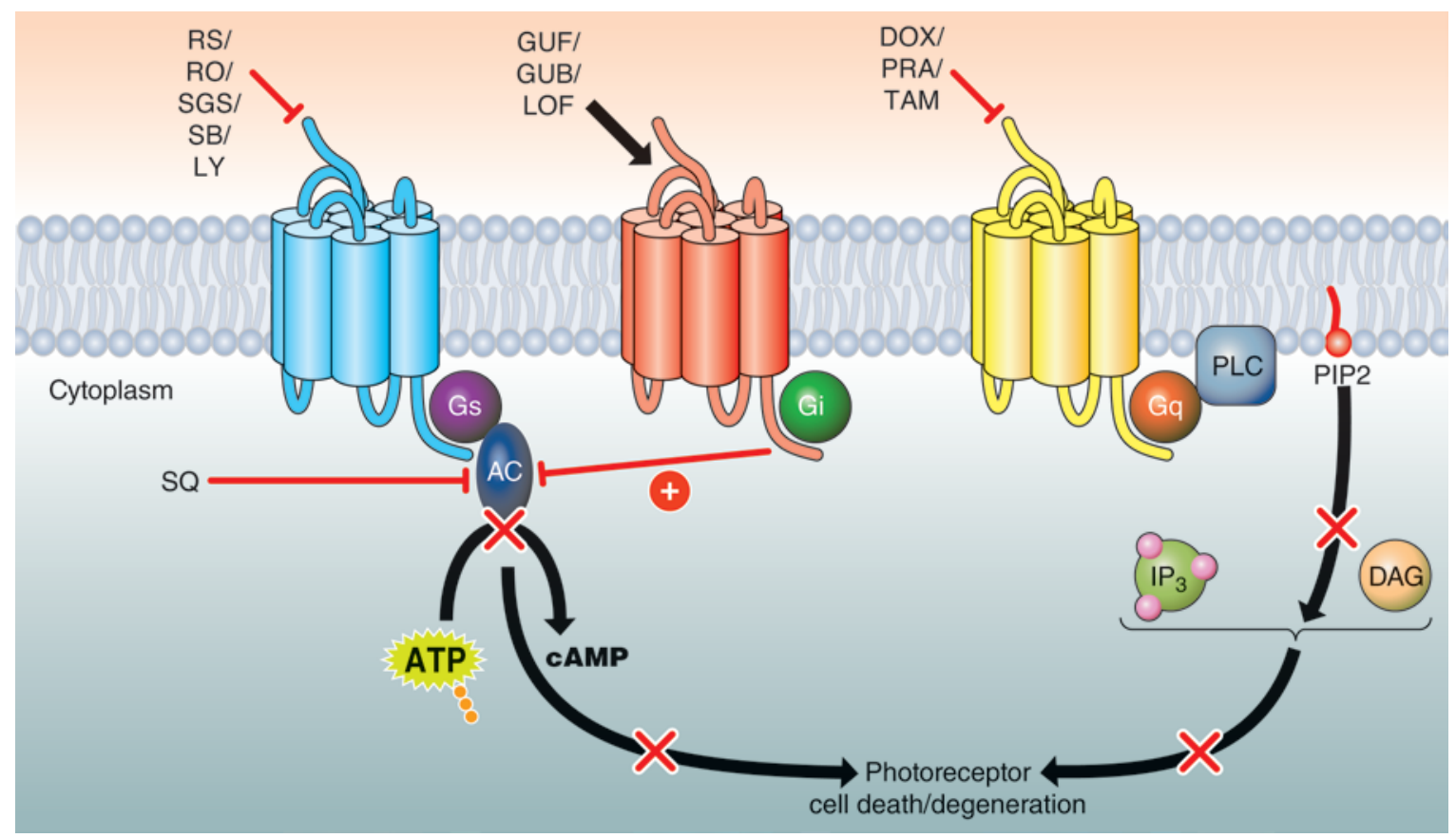

\section{Figure 12}

Systems pharmacological strategies targeting multiple GPCRs can prevent the development of light-induced photoreceptor degeneration. Antagonists of multiple Gs-coupled GPCRs prevented photoreceptor cell death (red bar, top left), implying that increased activity of Gs-coupled GPCRs with subsequent activation of AC (black arrow) could cause photoreceptor cell death. In contrast, pharmacological activation of $\alpha 2-A R s$, Gi-coupled GPCRs (black arrow, top middle) suppressed AC activity (red bar). Therefore, AC as the central player mediating Gs-coupled and Gi-coupled GPCR signaling could also serve as a direct therapeutic target to protect photoreceptors from bright light-induced degeneration. Consistent with this hypothesis, protection could also be achieved by directly inhibiting AC activity with an AC inhibitor (red bar, left middle). Additionally, consistent with our previous finding that Gq-coupled GPCRs participate in photoreceptor degeneration, inhibition of $\alpha 1-A R$, a Gq-coupled GPCR, also proved effective in protecting photoreceptors from light-induced degeneration (red bar, top right).

the untreated eye of the patient who required enucleation for a large ocular melanoma and was immediately placed in RNAlater. The experimental sample was obtained from a tumor-free hemiretina. The eye had no signs of inflammation or abnormal neovascularization of the iris or retina.

Eye and retinal tissue libraries were prepared as previously described $(47,83)$. Each mouse and human library was run on the Illumina Genome Analyzer IIx (Illumina) in the CWRU Genomics core facility using 36-79 single-end read lengths. The processed and raw FASTQ files from mouse were previously deposited in the Gene Expression Omnibus database (GEO accession numbers GSE38359 and GSE29752).

Chemicals. DOX was purchased from Selleckchem. LOF was obtained from Santa Cruz Biotechnology, Inc. PRA, TAM, RS, RO, SB, SGS, LY, GUB, GUF, IDA, and SQ were purchased from Tocris Bioscience. Agomelatine, nefazodone, eltoprazine, cyproheptadine, pizotifen, GR 125487, RS 39604, SB203186, SB 399885, tamsulosin, phenoxybenzamine, phentolamine, fexofenadine, tolterodine, ABT-724, PD-168077, and yohimbine were purchased from Sigma-Aldrich. The chemical structures of all the pharmacologic agents screened are included in Supplemental Table 3.

Mouse treatments. All experimental compounds were administered to mice by i.p. injection through a 28 -gauge needle 30 minutes prior to bright light exposure. Tested compounds and their doses were: DOX, $1 \mathrm{mg} / \mathrm{kg}$ body weight (BW), $2 \mathrm{mg} / \mathrm{kg} \mathrm{BW}, 3 \mathrm{mg} / \mathrm{kg} \mathrm{BW}$, and $10 \mathrm{mg} / \mathrm{kg} \mathrm{BW}$, respectively; PRA, 2 mg/kg BW; TAM, 2 mg/kg BW; RS, 20 mg/kg BW; RO, 30 mg/kg BW; SB, $30 \mathrm{mg} / \mathrm{kg} \mathrm{BW;} \mathrm{SGS,} 30 \mathrm{mg} / \mathrm{kg} \mathrm{BW;} \mathrm{LY,} 10 \mathrm{mg} / \mathrm{kg}$ BW; GUB, $0.5 \mathrm{mg} / \mathrm{kg} \mathrm{BW}, 1 \mathrm{mg} / \mathrm{kg} \mathrm{BW}, 1.5 \mathrm{mg} / \mathrm{kg}$, and $2 \mathrm{mg} / \mathrm{kg} \mathrm{BW}$, respectively; GUF, 2 mg/kg BW; LOF, 2 mg/kg BW; SQ1, 0.083 mg/kg BW; SQ2, 0.125 mg/kg
BW; SQ3, $0.25 \mathrm{mg} / \mathrm{kg} \mathrm{BW;} \mathrm{SQ4,} 0.5 \mathrm{mg} / \mathrm{kg}$ BW; and IDA, $2.5 \mathrm{mg} / \mathrm{kg} \mathrm{BW}$ and $5 \mathrm{mg} / \mathrm{kg} \mathrm{BW}$. All tested compounds were dissolved in DMSO before injection except IDA, which was dissolved in $0.9 \%$ saline.

Spectral domain optical coherence tomography. Noninvasive ultra-highresolution spectral domain optical coherence tomography (SD-OCT) (Bioptigen) was performed for in vivo imaging of mouse retinas. Mice were anesthetized with an i.p. injection of an anesthetic cocktail consisting of ketamine $(6 \mathrm{mg} / \mathrm{ml})$ and xylazine $(0.44 \mathrm{mg} / \mathrm{ml})$ diluted with $10 \mathrm{mM}$ sodium phosphate, pH 7.2, and $100 \mathrm{mM} \mathrm{NaCl}$ at a dose of $20 \mu \mathrm{l} / \mathrm{g}$ BW. Pupils were dilated with $1 \%$ tropicamide prior to SD-OCT imaging. Five frames of OCT images were acquired in the B-scan mode and averaged for image presentation and analysis. Retinal pathology was subsequently scored according to the following criteria: grade 0 , outer nuclear layer (ONL) was completely disrupted with no visible appearance observed; grade 1 , extensive disruption of an ONL spanning the retina $500 \mu \mathrm{m}$ away from the optic nerve head with an ONL thickness less than $0.01 \mathrm{~mm}$; grade 2, extensive disruption of the ONL spanning the retina $500 \mu \mathrm{m}$ away from the optic nerve head with a measured ONL thickness between 0.01 and $0.03 \mathrm{~mm}$; grade 3 , reduction in ONL thickness, with a measured thickness between 0.03 and $0.05 \mathrm{~mm}$; grade 4 , intact $\mathrm{ONL}$ with a measured thickness greater than $0.05 \mathrm{~mm}$. For evaluation of the impact of Gi and Gq pathways, data points were plotted as the percentage of eyes that developed significant retinal degeneration with an ONL thickness less than $0.035 \mathrm{~mm}$.

SLO imaging. SLO imaging (Heidelberg Engineering) was performed for in vivo whole-fundus imaging of mouse retinas (84). Mice were anesthetized by i.p. injection of the anesthetic cocktail indicated above, followed 
by pupil dilation with $1 \%$ tropicamide prior to SLO imaging in the autofluorescence mode. The numbers of autofluorescent spots were counted and subjected to statistical analyses as described below.

IHC. Retinal IHC was performed as previously described (85). Briefly, the eyes were enucleated and, after removal of the cornea, lens, and vitreous body, the eye cups were fixed in $4 \%$ paraformaldehyde and processed for cryosectioning. Twelve-micrometer-thick cryosections were cut, collected, and examined for rhodopsin expression, and PNA staining of the cone sheath and nuclear DAPI staining were performed.

ERGs. ERGs were performed as previously described (85). Briefly, darkadapted mice were examined under dim red light transmitted through a Kodak No. 1 Safelight Filter (transmittance $560 \mathrm{~nm}$ ). Pupils were dilated with $1 \%$ tropicamide after anesthesia induced by the method described above. Contact lens electrodes were placed on the eyes, and a reference electrode and ground electrode were positioned on the ear and tail, respectively. ERGs were recorded with the UTAS E-3000 universal testing and electrophysiologic system (LKC Technologies, Inc.).

In vivo detection of ROS. In vivo ROS generation was evaluated as previously described (39). The ROS probe DHE, at a dose of $20 \mathrm{mg} / \mathrm{kg} \mathrm{BW}$ in $25 \mu \mathrm{l}$ of DMSO, was administered to $A b c a 4^{-/-} R d h 8^{-/-}$mice via i.p. injection 1 hour before light exposure. Eye cups obtained after removing the cornea, lens, and vitreous body from enucleated eye globes 3 hours after light illumination were fixed in $4 \%$ paraformaldehyde. Cryosections were prepared from fixed eye cups and cut at $12-\mu \mathrm{m}$ thickness for microscopic assessment of ROS fluorescence in the retina using ImageJ software (NIH).

TPM imaging. Ten days after bright light exposure, TPM images were obtained as previously described (32). Briefly, we used a Leica TCS SP5 upright confocal microscope equipped with a 1.0-NA water immersion objective and tunable laser Vision S (Coherent) delivering 75-femtosecond laser pulses at an $80-\mathrm{MHz}$ pulse repetition frequency. Emission spectra were obtained with a TCS SP5 (Leica) spectrally sensitive detector in the descanned configuration. Only intact, freshly enucleated mouse eyes were used for imaging. Before enucleation, mice were anesthetized by i.p. injection of the anesthetic cocktail indicated above and then euthanized.

Quantification of selected drugs in mouse tissue. Six-week-old C57BL/6J WT mice (The Jackson Laboratory) were treated with DOX, GUB, or SQ at a single dose of 10, 2, and $0.5 \mathrm{mg} / \mathrm{kg}$, respectively. Each compound was dissolved in 50\% DMSO in PBS $(137 \mathrm{mM} \mathrm{NaCl}, 2.7 \mathrm{mM} \mathrm{KCl}, 0.67 \mathrm{mM}$ $\mathrm{Na}_{2} \mathrm{HPO}_{4} / \mathrm{KH}_{2} \mathrm{PO}_{4}, \mathrm{pH}$ 7.4) and administrated by i.p. injection. Thirty minutes later, the mice were anesthetized. Blood samples were collected using a cardiac puncture technique and subsequently centrifuged ( 5 minutes at $16,000 \mathrm{~g}$ ) for serum collection. Prior to harvesting eyes, the mice were intracardially perfused with PBS to minimize blood contamination. Eyeballs were immediately homogenized in $0.1 \mathrm{ml}$ of PBS. The homogenate and serum samples were spiked with 100 pmols of internal standard (IS) (PRA - 2-[4-(2-furoyl)piperazin-1-yl]-6,7-dimethoxyquinazolin-4-amine or clenbuterol - RS -1-(4-amino-3,5-dichlorophenyl)-2-(tert-butylamino) ethanol) (both from Sigma-Aldrich) for DOX, GUB, or SQ quantification, respectively $(86,87)$. Next, to precipitate excess proteins, $0.3 \mathrm{ml}$ of methanol was added to both the eye homogenate and $0.1 \mathrm{ml}$ of serum. Samples were vortexed for 30 seconds, followed by centrifugation for 15 minutes at $16,000 \mathrm{~g}$. Clear supernatants were collected and used directly for liquid chromatographic mass spectrometric (LC-MS) analysis.

MS-based detection and quantification of DOX, GUB, and SQ was performed with an LXQ linear ion trap mass spectrometer (Thermo Scientific) equipped with an electrospray ionization (ESI) interface and coupled to an Agilent 1100 HPLC (Agilent Technologies). Separation of drugs and the ISs was achieved on a reverse-phase C18 Phenomenex HPLC column $(250 \times 4.60 \mathrm{~mm} ; 5 \mu \mathrm{m})$ by a linear gradient of $0 \%$ to $100 \%$ acetonitrile in water within 15 minutes at a flow rate of $0.5 \mathrm{ml} /$ minute. All solvents contained
$0.1 \%$ formic acid $(\mathrm{v} / \mathrm{v})$. The HPLC effluent was sprayed into the mass spectrometer via an ESI probe operated in the positive ionization mode. Parameters of ionization and detection were tuned with synthetic standards for the drugs to achieve the highest possible sensitivity. DOX and PRA (IS) were detected by selected reaction monitoring (SRM) using $\mathrm{m} / \mathrm{z} 452.2 \rightarrow 344.2$ and $384.2 \rightarrow 247.1$ transitions, whereas GUB, SQ, and their corresponding IS clenbuterol were detected by fragmentation at $\mathrm{m} / \mathrm{z} 231.1 \rightarrow 214.0$, $206.1 \rightarrow 136.0$, and $277.1 \rightarrow 259.1$, respectively. The elution times for DOX, GUB, and SQ were approximately $12.8,12.3$, and 10.8 minutes, respectively. Both ISs eluted at 12.0 minutes (Figure 10). Calibration curves were calculated based on the linear relationship between ratios of the SRM ion intensity peak area corresponding to the selected drug and the IS versus molar ratios of the compounds in a range of 20 to $500 \mathrm{pmol}$ (Figure 10H).

Statistics. Results were collected from at least 4 mice per experimental group. Data are expressed as the means \pm SEM, and statistical analyses were performed using a 1-way Students $t$ test or ANOVA. A $P$ value of $\leq 0.05$ was considered statistically significant.

Study approval. All animal procedures and experiments were approved by the IACUC of CWRU and conformed to recommendations of both the American Veterinary Medical Association Panel on Euthanasia and the Association of Research for Vision and Ophthalmology. Clinical evaluation of the human patient from whom retinal tissue was obtained was performed at the Cleveland Clinic Cole Eye Institute (Cleveland, Ohio, USA), and written permission was obtained from the patient. This research conformed to the tenets of the Declaration of Helsinki and was conducted in accordance with approved protocols of the Cleveland Clinic Cole Eye Institute.

\section{Acknowledgments}

We thank Leslie T. Webster Jr. and members of Palczewski's laboratory for helpful comments on this manuscript and Mee Jee Lee (CWRU) for technical support. We also thank Yiyuan Yuan and John Feng for assays performed on C. elegans. We thank Hiroshi Maeno for siRNA experiments and Bhubannananda Sahu for ERG analyses. We would also like to thank Jonathan E. Sears (Cleveland Clinic Cole Eye Institute) for ophthalmic examination of the human patient and retinal tissue isolation. This work was supported by funding from the NIH (K08EY019031, to A. Maeda; K08EY019880, to T. Maeda; EY009339, to K. Palczewski; EY021126, to K. Palczewski; EY022658, to A. Maeda; AG043645, to G. Palczewska; and P30 EY11373). Support was also provided by the Research to Prevent Blindness Foundation, the Foundation Fighting Blindness, the Fight for Sight, the Ohio Lions Eye Research Foundation, and the Program for Professor of Special Appointment at Shanghai Institutions of Higher Learning (to Y. Chen). D. Mustafi was supported in part by the NIH CWRU Medical Scientist Training Program Grant (T32GM007250) and the Visual Sciences Training Grant (T32EY007157). K. Palczewski is a John H. Hord Professor of Pharmacology and CSO of Polgenix Inc.

Received for publication January 28, 2013, and accepted in revised form September 12, 2013.

Address correspondence to: Krzysztof Palczewski, Department of Pharmacology, School of Medicine, Case Western Reserve University, 10900 Euclid Avenue, Cleveland, Ohio 44160, USA. Phone: 216.368.4631; Fax: 216.368.1300; E-mail: kxp65@case.edu.

Yu Chen's present address is: Yueyang Hospital and Clinical Research Institute of Integrative Medicine, Shanghai University of TCM, Shanghai, China. 
1. Bamshad MJ, et al. Exome sequencing as a tool for Mendelian disease gene discovery. Nat Rev Genet. 2011;12(11):745-755

2. Ku CS, et al. Exome versus transcriptome sequencing in identifying coding region variants. Expert Rev Mol Diagn. 2012;12(3):241-251.

3. De Matteis MA, Luini A. Mendelian disorders of membrane trafficking. NEngl J Med. 2011;365(10):927-938.

4. Hubner $\mathrm{N}$, et al. Integrated transcriptional profiling and linkage analysis for identification of genes underlying disease. Nat Genet. 2005;37(3):243-253.

5. Dermitzakis ET. Cellular genomics for complex traits. Nat Rev Genet. 2012;13(3):215-220.

6. Cooper GM, Shendure J. Needles in stacks of needles: finding disease-causal variants in a wealth of genomic data. Nat Rev Genet. 2011;12(9):628-640.

7. Zhou K, Pearson ER. Insights from genome-wide association studies of drug response. Annu Rev Pharmacol Toxicol. 2013;53:299-310.

8. Kleeberger SR, Peden D. Gene-environment interactions in asthma and other respiratory diseases. Annu Rev Med. 2005;56:383-400.

9. de Krom M, Bauer F, Collier D, Adan RA, la Fleur SE. Genetic variation and effects on human eating behavior. Annu Rev Nutr. 2009;29:283-304.

10. Gutiu IA, Andries A, Mircioiu C, Radulescu F, Georgescu AM, Cioaca D. Pharmacometabonomics, pharmacogenomics and personalized medicine. Rom J Intern Med. 2010;48(2):187-191.

11. Camilleri $M$. The role of pharmacogenetics in nonmalignant gastrointestinal diseases. Nat Rev Gastroenterol Hepatol. 2012;9(3):173-184.

12. Stallmeyer B, et al. Mutational spectrum in the $\mathrm{Ca}(2+)$ - activated cation channel gene TRPM4 in patients with cardiac conductance disturbances. Hum Mutat. 2012;33(1):109-117.

13. Tremblay J, Hamet P. Genetics of pain, opioids, and opioid responsiveness. Metabolism. 2010; 59(suppl 1):S5-S8

14. Allerheiligen SR. Next-generation model-based drug discovery and development: quantitative and systems pharmacology. Clin Pharmacol Ther. 2010; 88(1):135-137.

15. Hansen J, Zhao S, Iyengar R. Systems pharmacology of complex diseases. Ann N Y Acad Sci. 2011;1245:E1-E5.

16. Zhao S, Iyengar R. Systems pharmacology: network analysis to identify multiscale mechanisms of drug action. Annu Rev Pharmacol Toxicol. 2012;52:505-521.

17. van der Greef J, McBurney RN. Innovation: Rescuing drug discovery: in vivo systems pathology and systems pharmacology. Nat Rev Drug Discov. 2005; 4(12):961-967.

18. Bai JP, Abernethy DR. Systems pharmacology to predict drug toxicity: integration across levels of biological organization. Annu Rev Pharmacol Toxicol. 2013;53:451-473.

19. Kassahn KS, Waddell N, Grimmond SM. Sequencing transcriptomes in toto. Integr Biol (Camb). 2011; 3(5):522-528.

20. Costa V, Angelini C, De Feis I, Ciccodicola A. Uncovering the complexity of transcriptomes with RNA-Seq. J Biomed Biotechnol. 2010;2010:853916.

21. Provis JM. Development of the primate retinal vasculature. Prog Retin Eye Res. 2001;20(6):799-821.

22. Fruttiger M. Development of the retinal vasculature. Angiogenesis. 2007;10(2):77-88.

23. Travis GH, Golczak M, Moise AR, Palczewski K Diseases caused by defects in the visual cycle: retinoids as potential therapeutic agents. Annu Rev Pharmacol Toxicol. 2007;47:469-512.

24. Mustafi D, Engel AH, Palczewski K. Structure of cone photoreceptors. Prog Retin Eye Res. 2009; 28(4):289-302.

25. Palczewski K. Chemistry and biology of vision. J Biol Chem. 2012;287(3):1612-1619.

26. Castro Lima V, Rodrigues EB, Nunes RP, Sallum JF, Farah ME, Meyer CH. Simultaneous confocal scan- ning laser ophthalmoscopy combined with high-resolution spectral-domain optical coherence tomography: a review. J Ophthalmol. 2011;2011:743670.

27. Costa RA, et al. Retinal assessment using optical coherence tomography. Prog Retin Eye Res. 2006; 25(3):325-353.

28. Gabriele ML, et al. Three dimensional optical coherence tomography imaging: advantages and advances. Prog Retin Eye Res. 2010;29(6):556-579.

29. Imanishi Y, Batten ML, Piston DW, Baehr W, Palczewski K. Noninvasive two-photon imaging reveals retinyl ester storage structures in the eye. J Cell Biol. 2004;164(3):373-383.

30. Hunter JJ, et al. Images of photoreceptors in living primate eyes using adaptive optics two-photon ophthalmoscopy. Biomed Opt Express. 2010;2(1):139-148.

31. Maeda A, et al. Primary amines protect against retinal degeneration in mouse models of retinopathies. Nat Chem Biol. 2012;8(2):170-178.

32. Palczewska G, et al. Noninvasive multiphoton fluorescence microscopy resolves retinol and retinal condensation products in mouse eyes. Nature Medicine. 2010;16(12):1444-1449.

33. Orban T, Palczewska G, Palczewski K. Retinyl ester storage particles (retinosomes) from the retinal pigmented epithelium resemble lipid droplets in other tissues. J Biol Chem. 2011;286(19):17248-17258.

34. Tsybovsky Y, Molday RS, Palczewski K. The ATP-binding cassette transporter ABCA4: structural and functional properties and role in retinal disease. Adv Exp Med Biol. 2010;703:105-125.

35. Kiser PD, Golczak M, Maeda A, Palczewski K. Key enzymes of the retinoid (visual) cycle in vertebrate retina. Biochim Biophys Acta. 2012;1821(1):137-151.

36. Maeda A, Golczak M, Maeda T, Palczewski K. Limited roles of Rdh8, Rdh12, and Abca 4 in alltrans-retinal clearance in mouse retina. Invest $\mathrm{Oph}$ thalmol Vis Sci. 2009;50(11):5435-5443

37. Maeda A, Maeda T, Golczak M, Palczewski K. Retinopathy in mice induced by disrupted all-trans-retinal clearance. J Biol Chem. 2008;283(39):26684-26693.

38. Maeda A, et al. Involvement of all-trans-retinal in acute light-induced retinopathy of mice. J Biol Chem. 2009;284(22):15173-15183.

39. Chen Y, et al. Mechanism of all-trans-retinal toxicity with implications for stargardt disease and age-related macular degeneration. J Biol Chem. 2012; 287(7):5059-5069.

40. Lem J, et al. Morphological, physiological, and biochemical changes in rhodopsin knockout mice. Proc Natl Acad Sci U S A. 1999;96(2):736-741.

41. Kohno H, et al. Photoreceptor proteins initiate microglial activation via Toll-like receptor 4 in retinal degeneration mediated by all-trans-retinal. J Biol Chem. 2013;288(21):15326-15341.

42. Buck K, Voehringer P, Ferger B. The $\alpha(2)$ adrenoceptor antagonist idazoxan alleviates L-DOPA-induced dyskinesia by reduction of striatal dopamine levels: an in vivo microdialysis study in 6-hydroxydopaminelesioned rats. J Neurochem. 2010;112(2):444-452.

43. Jeon CJ, Strettoi E, Masland RH. The major cell populations of the mouse retina. J Neurosci. 1998; 18(21):8936-8946.

44. Sparrow JR, Wu Y, Kim CY, Zhou J. Phospholipid meets all-trans-retinal: the making of RPE bisretinoids. J Lipid Res. 2010;51(2):247-261.

45. Cideciyan AV, et al. ABCA4 disease progression and a proposed strategy for gene therapy. Hum Mol Genet. 2009;18(5):931-941.

46. Sparrow JR, et al. The bisretinoids of retinal pigment epithelium. Prog Retin Eye Res. 2012; 31(2):121-135

47. Mustafi D, Maeda T, Kohno H, Nadeau JH, Palczewski K. Inflammatory priming predisposes mice to age-related retinal degeneration. J Clin Invest. 2012;122(8):2989-3001

48. Ambati J, Fowler BJ. Mechanisms of age-related macular degeneration. Neuron. 2012;75(1):26-39.
49. von Lintig J, Kiser PD, Golczak M, Palczewski K. The biochemical and structural basis for trans-tocis isomerization of retinoids in the chemistry of vision. Trends Biochem Sci. 2010;35(7):400-410.

50. Kiser PD, Palczewski K. Membrane-binding and enzymatic properties of RPE65. Prog Retin Eye Res. 2010;29(5):428-442.

51. Yu DY, Cringle SJ. Oxygen distribution and consumption within the retina in vascularised and avascular retinas and in animal models of retinal disease. Prog Retin Eye Res. 2001;20(2):175-208.

52. Lange CA, Bainbridge JW. Oxygen sensing in retinal health and disease. Ophthalmologica. 2012; 227(3):115-131.

53. Bazan NG. Cell survival matters: docosahexaenoic acid signaling, neuroprotection and photoreceptors. Trends Neurosci. 2006;29(5):263-271.

54. Chertov AO, et al. Roles of glucose in photoreceptor survival. J Biol Chem. 2011;286(40):34700-34711.

55. Jones BW, et al. Retinal remodeling triggered by photoreceptor degenerations. J Comp Neurol. 2003;464(1):1-16.

56. McBee JK, Palczewski K, Baehr W, Pepperberg DR. Confronting complexity: the interlink of phototransduction and retinoid metabolism in the vertebrate retina. Prog Retin Eye Res. 2001;20(4):469-529.

57. London A, Benhar I, Schwartz M. The retina as a window to the brain-from eye research to CNS disorders. Nat Rev Neurol. 2012;9(1):44-53.

58. Nguyen-Legros J, Hicks D. Renewal of photoreceptor outer segments and their phagocytosis by the retinal pigment epithelium. Int Rev Cytol. 2000; 196:245-313.

59. Kevany BM, Palczewski K. Phagocytosis of retinal rod and cone photoreceptors. Physiology (Bethesda). 2010;25(1):8-15.

60. Fong WG, Tsilfidis C. Retinal degeneration and cellular suicide. Adv Exp Med Biol. 2012;723:207-214.

61. Mitter SK, et al. Autophagy in the retina: a potential role in age-related macular degeneration. $A d v$ Exp Med Biol. 2012;723:83-90.

62. Grimm C, Wenzel A, Williams T, Rol P, Hafezi F, Reme C. Rhodopsin-mediated blue-light damage to the rat retina: effect of photoreversal of bleaching. Invest Ophthalmol Vis Sci. 2001;42(2):497-505.

63. Wilt TJ, MacDonald R. Doxazosin in the treatment of benign prostatic hypertrophy: an update. Clin Interv Aging. 2006;1(4):389-401.

64. Doggrell SA. After ALLHAT: doxazosin for the treatment of benign prostatic hyperplasia. Expert Opin Pharmacother. 2004;5(9):1957-1964.

65. Kyprianou N, Litvak JP, Borkowski A, Alexander $\mathrm{R}$, Jacobs SC. Induction of prostate apoptosis by doxazosin in benign prostatic hyperplasia. J Urol. 1998;159(6):1810-1815

66. Dunn CJ, Matheson A, Faulds DM. Tamsulosin: a review of its pharmacology and therapeutic efficacy in the management of lower urinary tract symptoms. Drugs Aging. 2002;19(2):135-161.

67. Roehrborn CG. Efficacy of $\alpha$-adrenergic receptor blockers in the treatment of male lower urinary tract symptoms. Rev Urol. 2009;11(suppl 1):S1-S8.

68. Suzuki F, et al. Distribution of alpha-1 adrenoceptor subtypes in RNA and protein in rabbit eyes. $\mathrm{BrJ}$ Pharmacol. 2002;135(3):600-608.

69. Woldemussie E, Wijono M, Pow D. Localization of alpha 2 receptors in ocular tissues. Vis Neurosci. 2007;24(5):745-756.

70. Jerie P. Clinical experience with guanfacine in longterm treatment of hypertension. Br J Clin Pharmacol. 1980;10(suppl 1):37S-47S.

71. Jerie P. Clinical experience with guanfacine in longterm treatment of hypertension. Part II: adverse reactions to guanfacine. Br J Clin Pharmacol. 1980; 10(suppl 1):157S-164S

72. Walsh SL, Strain EC, Bigelow GE. Evaluation of the effects of lofexidine and clonidine on naloxone-precipitated withdrawal in opioid-dependent 
humans. Addiction. 2003;98(4):427-439.

73. Abdel-Majid RM, Tremblay F, Baldridge WH. Localization of adenylyl cyclase proteins in the rodent retina. Brain Res Mol Brain Res. 2002;101(1-2):62-70.

74. Cao P, et al. Light-sensitive coupling of rhodopsin and melanopsin to $G(i / o)$ and $G(q)$ signal transduction in Caenorhabditis elegans. FASEB J. 2012;26(2):480-491.

75. Eglen RM, et al. RS 23597-190: a potent and selective 5-HT4 receptor antagonist. Br J Pharmacol. 1993;110(1):119-126.

76. Sharif NA, Senchyna M. Serotonin receptor subtype mRNA expression in human ocular tissues, determined by RT-PCR. Mol Vis. 2006;12:1040-1047.

77. Michaels KV, et al. Serotonin receptor expression in the rodent retina. Presented at: ARVO 2011 Visionary Genomics; May 1-5, 2011; Fort Lauderdale, Florida, USA. Abstract 919/A111.
78. Collier RJ, et al. Agonists at the serotonin receptor $(5-\mathrm{HT}(1 \mathrm{~A}))$ protect the retina from severe photo-oxidative stress. Invest Ophthalmol Vis Sci. 2011;52(5):2118-2126.

79. Gilman AG. G proteins: transducers of receptor-generated signals. Annu Rev Biochem. 1987;56:615-649.

80. Grimm C, Wenzel A, Hafezi F, Yu S, Redmond TM, Reme CE. Protection of Rpe65-deficient mice identifies rhodopsin as a mediator of light-induced retinal degeneration. Nat Genet. 2000;25(1):63-66.

81. Hao W, et al. Evidence for two apoptotic pathways in light-induced retinal degeneration. Nat Genet. 2002;32(2):254-260.

82. Jacobson SG, McInnes RR. Blinded by the light. Nat Genet. 2002;32(2):215-216.

83. Mustafi D, et al. Defective photoreceptor phagocytosis in a mouse model of enhanced S-cone syndrome causes progressive retinal degeneration.
FASEB J. 2011;25(9):3157-3176.

84. Huber G, et al. Spectral domain optical coherence tomography in mouse models of retinal degeneration. Invest Ophthalmol Vis Sci. 2009;50(12):5888-5895.

85. Maeda A, et al. Role of photoreceptor-specific retinol dehydrogenase in the retinoid cycle in vivo. J Biol Chem. 2005;280(19):18822-18832.

86. Erceg M, Cindric M, Pozaic Frketic L, Vertzoni M, Cetina-Cizmek B, Reppas C. A LC-MS-MS method for determination of low doxazosin concentrations in plasma after oral administration to dogs. J Chromatogr Sci. 2010;48(2):114-119.

87. Harkins JD, et al. The detection and biotransformation of guanabenz in horses: a preliminary report. Vet Ther. 2003;4(2):197-209.

88. Palczewski K, Orban T. From atomic structures to neuronal functions of g protein-coupled receptors. Annu Rev Neurosci. 2013;36:139-164. 